This is the post-print version of the following article: Zheng, G; He, J; Kumar, V; Wang, S; Pastoriza-Santos, I; Pérez-Juste, J; Liz-Marzán, LM; Wong, K., Discrete metal nanoparticles with plasmonic chirality, Chem. Soc. Rev., 2021, Advance Article

DOI: $\underline{10.1039 / C 9 C S 00765 B}$

This article may be used for non-commercial purposes in accordance with RSC Terms and Conditions for Self-Archiving. 


\title{
Discrete metal nanoparticles with plasmonic chirality
}

\author{
Guangchao Zheng ${ }^{\mathrm{a}, \mathrm{b}}$, Jijun $\mathrm{He}^{\mathrm{c}}$, Vished Kumard, Shenli Wange, Isabel Pastoriza-Santos ${ }^{\mathrm{f}, \mathrm{g}}$, \\ Jorge Pérez-Juste ${ }^{\mathrm{f}, \mathrm{g*}}$, Luis M. Liz-Marzán ${ }^{\mathrm{d}, \mathrm{h}, \mathrm{i} *}$, Kwok-Yin Wong ${ }^{\mathrm{b} *}$ \\ a. School of Physics and Microelectronics, Zhengzhou University, Zhengzhou, 450001, P.R. \\ China \\ b. State Key Laboratory of Chemical Biology and Drug Discovery, Department of Applied \\ Biology and Chemical Technology, The Hong Kong Polytechnic University, Hunghom, Kowloon, \\ Hong Kong, China. Email: kwok-yin.wong@polyu.edu.hk \\ c. Institute of Physics, Ecole Polytechnique Federal de Lausanne, CH-1015, Lausanne, \\ Switzerland \\ d. CIC biomaGUNE, Basque Research and Technology Alliance (BRTA) Paseo de Miramón 182, \\ 20014 Donostia - San Sebastian, Spain.E-mail: Ilizmarzan@cicbiomagune.es \\ e. School of Food Science and Engineering, Henan University of Technology, LianHua Road 100, \\ Zhengzhou 450001, P. R. China \\ f. CINBIO, Universidade de Vigo, Departamento de Química Física, Campus Universitario As \\ Lagoas, Marcosende, 36310 Vigo, Span.Email: juste@uvigo.es \\ g. Galicia Sur Health Research Institute (IIS Galicia Sur), SERGAS-UVIGO, 36310 Vigo, Spain \\ h. Ikerbasque, Basque Foundation for Science, 48013 Bilbao, Spain \\ i. Centro de Investigación Biomédica en Red, Bioingeniería, Biomateriales y Nanomedicina, \\ CIBER-BBN, Paseo de Miramón 182, 20014 Donostia-San Sebastián, Spain
}

\section{Key Learning Points}

$\checkmark$ Optical activity in the visible and near-IR can be achieved from chiral nanostructures

$\checkmark$ The origin of the plasmonic chirality can be explained through theoretical modeling

$\checkmark$ Discrete metal nanoparticles have been synthesized by combinations of metal nanoparticles and chiral molecules, as well as by chiral growth in 3D

$\checkmark$ Discrete nanoparticles with plasmonic chirality have applications in various fields such as chemical sensing, circularly polarized photocatalysis, and photodynamic therapy 


\section{Abstract}

From a geometrical perspective, a chiral object does not have mirror planes or inversion symmetry. It exhibits the same physical properties as its mirror image (enantiomer), except for the chiroptical activity, which is often the opposite. Recent advancements have identified particularly interesting implications of chirality on the optical properties of metal nanoparticles, which are intimately related to localized surface plasmon resonance phenomena. Although such resonances are usually independent of the circular polarization of light, specific strategies have been applied to induce chirality, both in assemblies and at the single-particle level. In this tutorial review, we discuss the origin of plasmonic chirality, as well as theoretical models that have been proposed to explain it. We then summarise recent developments in the synthesis of discrete nanoparticles with plasmonic chirality by means of wet-chemistry methods. We conclude with a discussion of promising applications for discrete chiral nanoparticles. We expect this tutorial review to be of interest to researchers from a wide variety of disciplines where chiral plasmonics can be exploited at the nanoparticle level, such as chemical sensing, photocatalysis, photodynamic or photothermal therapies, etc.

\section{Introduction}

Chirality is present in our lives at all lengths scales, from the shape of galaxies to the conformation of single molecules. This term, derived from the Greek word for hand (kheir), refers to any object (in the context of chemistry commonly to a molecule) which is not superimposable onto its mirror image. As a peculiar example, the pods of green beans prefer to fold into chiral helical structures (Figure $1 \mathrm{~A}$ ). Although enantiomers (non-superimposable objects which are mirror images of each other) commonly show similar physical properties, their optical activities, defined as the ability of a substance to rotate the plane of polarization of a beam of light that is passed through it, are usually opposite. A chiral object will interact differently with left-handed and right-handed circularly polarized light at its specific absorption energy. Based on these premises, a circular dichroism (CD) spectrum results from the differential extinction produced in the chiral object and it allows to discern its conformational structure. Many 
biologically active molecules, such as amino acids, proteins, or DNA, exhibit CD in the ultraviolet (UV) region of the electromagnetic spectrum. The electronic and vibrational excitations of their chiral secondary structures are responsible for the differential absorption observed when illuminated with right- and left-handed circularly polarized light. CD spectroscopy is thus a widely used tool for studying the structure of biomolecules. Notwithstanding, natural molecules often display weak chiroptical responses. Additionally, the optical activity could be converted into a dimensionless $g$-factor by dividing the differential absorbance of circularly polarized light by the absorbance at the same wavelength. On the other hand, noble metal nanoparticles (NPs) can support localized surface plasmon resonances (LSPRs), collective and coherent oscillations of their conduction electrons confined at the metal-dielectric interface, upon excitation with an electromagnetic radiation. This optical phenomenon allows the confinement and manipulation of electrons at the nanoscale. The excitation of LSPRs generates highly intense electromagnetic fields at the nanoparticle surface, which can strongly interact with molecules located nearby, giving rise to a variety of phenomena such as surface-enhanced Raman scattering (SERS), surfaceenhanced infrared absorption (SEIRA), plasmon-enhanced luminescence, or plasmon-enhanced CD. Thus, the combination of chiral molecules with a weak CD response and achiral plasmonic nanomaterials can give rise to a hybrid nanostructure with enhanced and tailored chiroptical properties.

Additionally, it has been reported that chiral plasmonic nanostructures can also be created through the synthesis of particles with chiral morphology (dissymmetric shape), or by assembling achiral plasmonic nanoparticles into chiral configurations. The latter are often mediated by chiral molecular templates such as DNA, ${ }^{1}$ proteins, ${ }^{2}$ or polymeric fibers, ${ }^{3}$ among others ${ }^{4}$ (selected examples are shown in Figure 1). Regardless of the methodology employed for their fabrication, chiral plasmonic nanostructures exhibit significantly larger optical activity than molecules do. Particularly interesting are the light-manipulating capabilities that chiral nanomaterials can offer as circular polarizers, in 
enantioselective-based sensing, enantiomeric separation, and photocatalytic or photothermal chirality.

Typically, chiral plasmonic nanostructures are synthesized either by means of topdown nanofabrication techniques, such as lithography, or through the directedassembly of achiral plasmonic NPs. Unfortunately, these approaches pose significant drawbacks, such as limitations toward large-scale production, or limited control over the dimensions and uniformity of self-assembled nanostructures and their subsequent manipulation toward applications. 
A

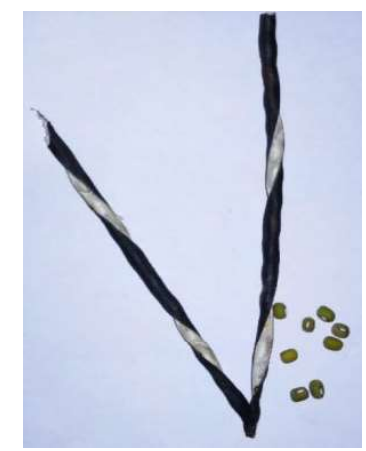

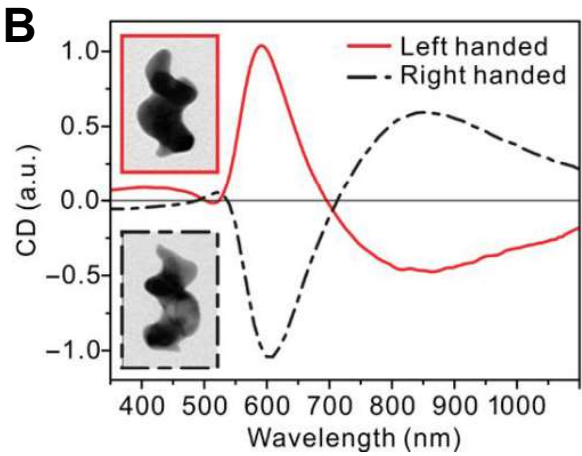

Left-handed helix Right-handed helix

C

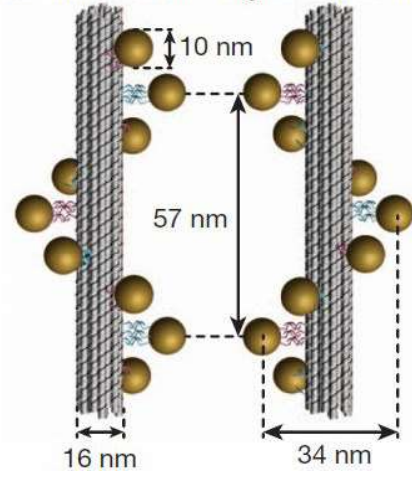

D

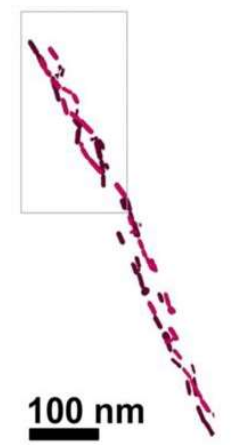

E

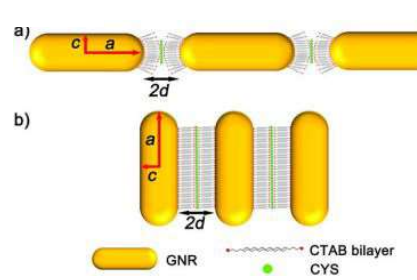

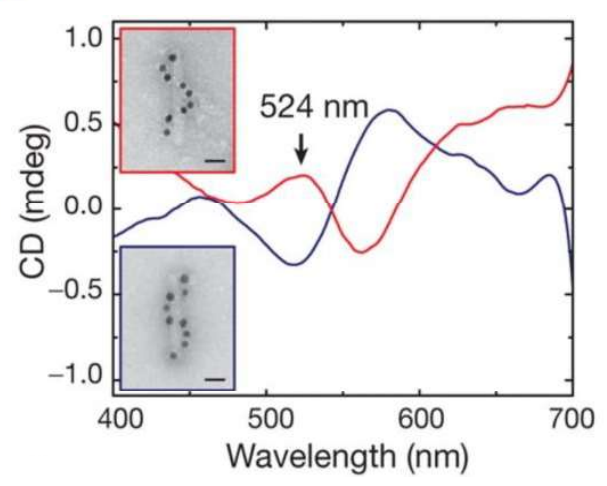
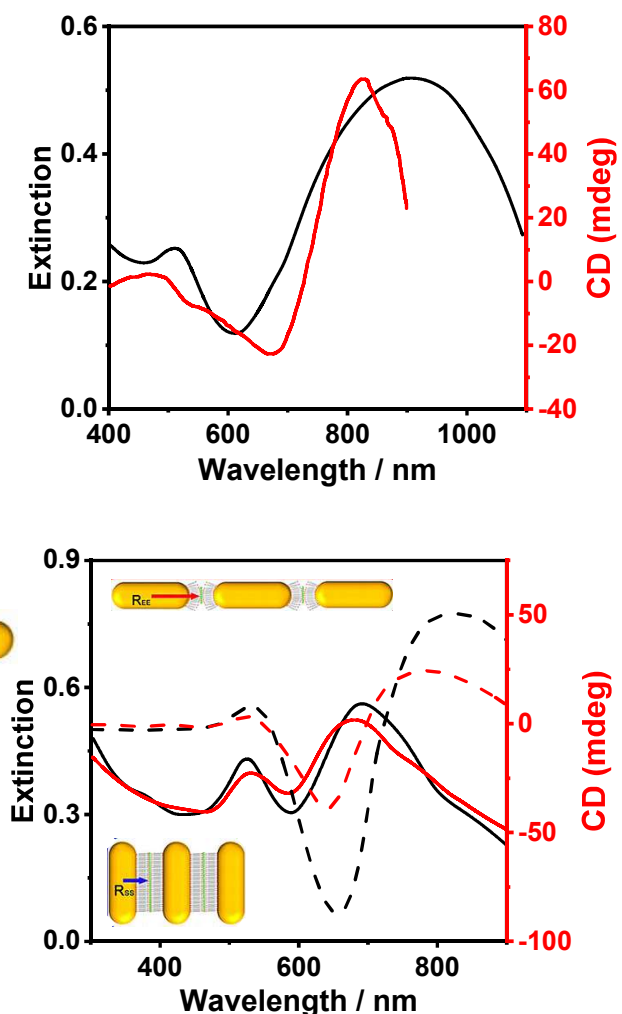

Figure 1. Examples of chiral (nano)structures. (A) Photograph of chiral pods of green beans. (B) Chiroptical response of Au nanohelices fabricated by lithography. The insets show representative TEM images of the helices. Adapted from ref. 5. Copyright 2013, Macmillan Publishers Ltd: Nature Materials. (C) Left: Scheme of nanohelices obtained via 
DNA origami-directed self-assembly of $10 \mathrm{~nm}$ Au nanoparticles. Right: Circular dichroism of left-handed (red line) and right-handed (blue line) nanohelices. The insets show representative TEM images. Adapted from ref. 6. Copyright 2012, Macmillan Publishers Ltd: Nature (D) Left: Cryo-TEM tomography reconstruction of a 3D chiral arrangement of Au nanorods on an $\alpha$-synuclein fibril and enlarged view of the top part of the assembly. Right: extinction and CD spectra of a colloidal dispersion of Au nanorod/ $\alpha$-synuclein selfassembled fibers. Adapted from ref. 2. Copyright 2018, National Academy of Sciences. (E) Left: Diagram of Au nanorods in end-to-end, EE, (a) or side-to-side, SS, (b) assemblies, mediated by cysteine and CTAB. Right: Comparison of the optical properties of EE (black lines) and SS (red lines) assembled Au nanorods. Adapted from ref. 7. Copyright 2014, American Chemical Society.

Alternatively, these drawbacks can be circumvented by synthesising discrete plasmonic nanoparticles with dissymmetric morphology or through plasmonic nanoparticle-chiral molecule complexes. We present in this tutorial review the fundamentals of theoretical models that have been developed to understand the mechanism of plasmon-induced chirality, together with the most relevant methods for the synthesis of discrete chiral plasmonic nanoparticles and proposed strategies for their application in various fields. Although excellent reviews have been published regarding the fabrication, application, and theoretical understanding of chiral plasmonic nanostructures, most of them are devoted to nanostructures fabricated by molecular directed-assembly technologies. ${ }^{8}$ Given the recent but fast development of the field, we decided to focus on wet-chemical approaches toward the synthesis of plasmonic nanoparticles with intrinsic chiroptical features, and their potential applications in the fields of enantioselective sensing and photocatalytic or photothermal chiral activity.

\section{The origin of plasmonic chirality.}

In general, a chiral object is defined as one which cannot be superimposed with its mirror image. In Chemistry, molecular chirality is commonly caused by a disymmetrical carbon atom, i.e. a carbon atom bonded to four different chemical groups or substituents. Notwithstanding, chirality and optical activity can also be found in a number of molecules which do not contain chiral carbon atoms. 
Examples include hexahelicene and ortho-condensed polycyclic aromatic compounds, which display a helical molecular structure, giving rise to chirality and circular dichroism. These chiral molecules possess delocalized electron orbitals that extend over the entire structure. It should be noted that the optical activity of most chiral molecules is rather weak, due to their small extinction crosssection. Therefore, the applicability of CD spectroscopy to characterize the secondary structure (i.e., the types of molecular bonds), conformation and dynamics of biomolecules and macromolecules (i.e., the orientation of orbitals) requires either high concentrations or large volumes. In general, two models have been proposed to describe chiroptical activity in molecular systems, namely the helical model and the coupling of two mirror-symmetrically arranged electric dipoles. ${ }^{9,} 10$

At the nanoscale, LSPRs in plasmonic nanoparticles enable the confinement of light and the generation of strong electromagnetic near fields, which might lead to enhanced molecule-metal chiroptical interactions. Numerous numerical and analytical theoretical models have been extensively developed to predict and understand the optical properties of plasmonic nanostructures, based on the resolution of Maxwell's equations. ${ }^{11,} 12$ Notwithstanding, plasmon-induced CD has been less studied and a complete understanding of this phenomenon would be necessary for an optimized development of efficient molecular-plasmonic systems. We provide herein a summary of state-of-the-art theoretical models to describe plasmonic effects on the CD response of chiral molecules. Considering the size of plasmonic NPs, the effect of nanoplasmonics can be categorized as; (a) near-field induction model (dipole-dipole interactions) for nanoparticles with sizes much smaller than the wavelength of the incident light, i.e. $a_{\text {metal }} \ll \lambda$, and (b) far-field induction model (electrodynamic interaction with retardation effect) for nanoparticles with $\mathrm{a}_{\text {metal }} \sim \lambda .^{13}$

The first model can be exemplified in a simple system comprising a chiral molecule and a spherical plasmonic NP, as shown in Figure $2 A .{ }^{14}$ In this molecule-NP complex, the chiral molecule is presented by electrical and magnetic dipole moments, $\boldsymbol{\mu}$ and $\mathbf{m}$, respectively. The angle formed between $\boldsymbol{\mu}$ and $\mathbf{m}$ inside the 
chiral molecule can be influenced by the NP's LSPR, upon excitation with light.. Besides, the complex will show a new plasmon-induced CD peak at the LSPR wavelength produced by the chiral current generated inside the plasmonic NP via Coulomb dipole-dipole interaction. ${ }^{14}$ Therefore, the optical activity of chiral plasmonic nanostructures is determined by the nanoparticle size, the nature of the nanoparticle and the molecule, and the molecule-NP distance, R. When R is small, the Coulomb dipole-dipole interaction dominates the coupling between molecule and NP. Under this condition, the CD signal of the molecule-NP system $\left(C D_{\text {total }}\right)$ can be described as a sum of two terms:

$\mathrm{CD}_{\text {total }}=\mathrm{CD}_{\text {molecule }}+\mathrm{CD}_{\text {plasmon }}$

where $C D_{\text {molecule }}$ and $C D_{\text {plasmon }}$ are the $C D$ signal of the molecule and $N P$, respectively. $C D_{\text {molecule }}$ can be further expressed as:

$\mathrm{CD}_{\text {molecule }}=E_{0}^{2} \frac{8}{3} \sqrt{\varepsilon_{0} \omega_{0}} \frac{\Gamma_{12}}{\left|\hbar \omega-\hbar \omega_{0}+i \Gamma_{12}\right|^{2}} \operatorname{Im}\left[\left(\hat{P} \cdot \boldsymbol{\mu}_{12}\right) \cdot \boldsymbol{m}_{21}\right]=a(\omega) \cdot \operatorname{Im}$ $\left[\left(\hat{P} \cdot \boldsymbol{\mu}_{12}\right) \cdot \boldsymbol{m}_{21}\right]$

where $E_{0}$ is the electric field of the incident light, $\varepsilon_{0}$ is the dielectric constant of the medium, $\omega_{0}$ is the frequency of the molecular absorption, $\Gamma_{12}$ is the broadening of the molecular transition from the ground state to the excited state, and $\hat{P}$ is a tensor describing the plasmon-enhanced factor of the electric field. ${ }^{14}$, 15 The overall coefficient $a(\omega)$ indicates the absorption transition profile of the chiral molecule from the ground state $|1\rangle$ to the excited state $|2\rangle$. Equation (2) clearly indicates that the chiral response of the molecule becomes larger as the plasmon-enhancement effect increases. This term reflects the plasmonenhancement effect on the transition of the chiral molecule, which produces a strong CD signal over the wavelength range of the molecular transition, see Figure 2 B. ${ }^{15}$ 
A

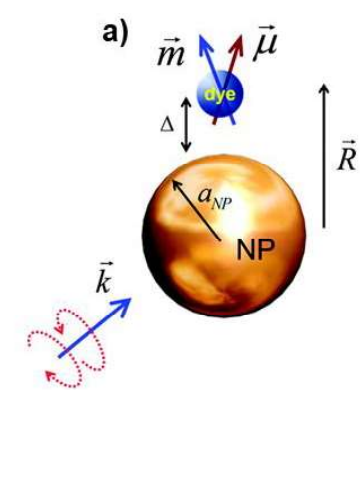

b)

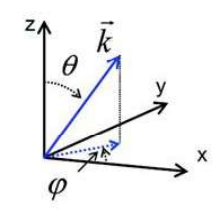

c)

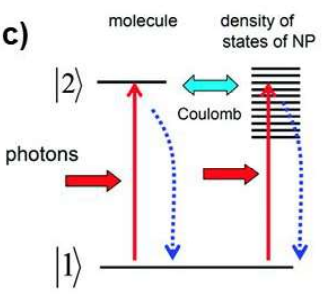

B d)

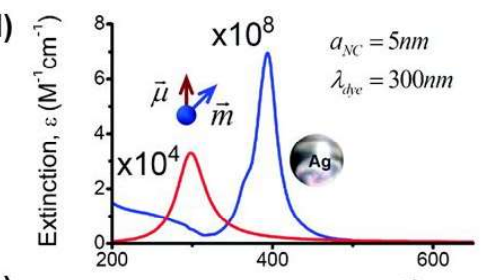

e)

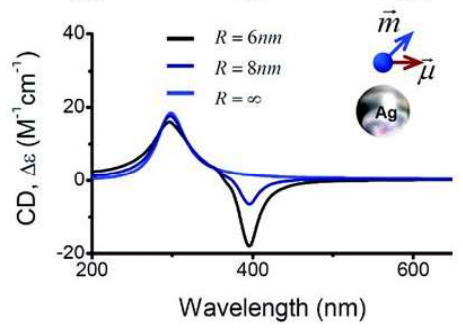

C

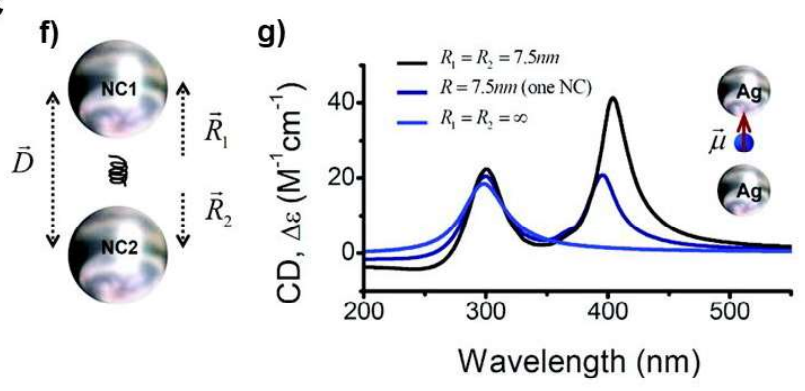

D

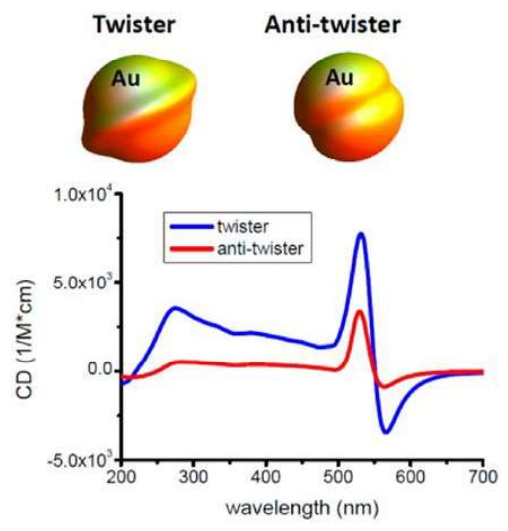

Figure 2. (A) Scheme of a complex made of a metal nanoparticle and a dye molecule (a).

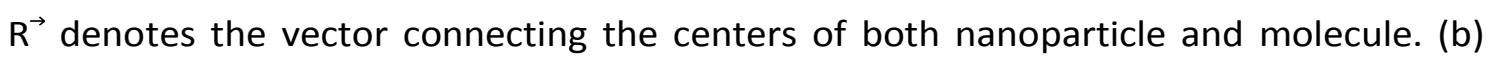
System of coordinates and the definition of polar angles used in the formulation. (c) Quantum transitions in the system; the solid vertical (horizontal) arrows represent light (Coulomb)-induced transitions. The dotted vertical arrows are the relaxation processes. Reproduced from ref. 14. Copyright 2010, American Chemical Society. (B) Complex made of an Ag NP and a dye molecule. (d) Extinction spectra of a molecule alone and a Au NP alone. (e) Calculated CD spectra for Ag NP-molecule complexes with three different molecule-NP distances, R, as indicated. Reproduced from ref. 15. Copyright 2011, American Chemical Society. (C) Model of a system made of a NP dimer and a chiral molecule (f). (e) Calculated CD spectra of a dimer-molecule complex for $R_{1}=R_{2}=7.5 \mathrm{~nm}$ and for $R_{1}=R_{2}=\infty$, being $R_{1}$ and $R_{2}$ the distances between the molecule and each NP in the dimer. The calculated CD spectrum for a single NP and $R=7.5 \mathrm{~nm}$ is also shown. Reproduced from ref. 15. Copyright 2011, American Chemical Society. (D) Scheme of a right-handed twister and an antitwister Au NP with an average radius $\left(R_{0}\right)$ of $7 \mathrm{~nm}$ and amplitude of radius variations $(\delta R)$ of 1.5 and -0.8 , respectively, together with the 
calculated CD response for both chiral Au NPs. Reproduced from ref. 16. Copyright 2012, American Chemical Society.

The second contribution term to the $C D$ signal in eq. 1 is the plasmon-induced $C D$, which is associated with the dissipation of light inside the plasmonic component. Considering a simple molecular-plasmonic system with spherical geometry, ${ }^{13}$ the plasmon-induced CD can be described as

$$
\begin{gathered}
\operatorname{CD}_{\text {plasmon }}=\operatorname{Im}\left(\varepsilon_{\text {metal }}\right) \frac{8 r_{\text {metal }}^{3}}{9 \varepsilon_{0} R^{3}} E_{0}^{2} \sqrt{\varepsilon_{0}}\left|\frac{3 \varepsilon_{0}}{\left(2 \varepsilon_{0}+\varepsilon_{\text {metal }}\right)}\right|^{2} \\
\operatorname{Im}\left[\frac{\left(\mu_{12 x} \cdot m_{21 x}+\mu_{12 y} \cdot m_{21 y}+\mu_{12 y} \cdot m_{21 x}\right)}{\hbar \omega-\hbar \omega_{0}+i \Gamma_{12}}\right]
\end{gathered}
$$

where $\varepsilon_{\text {metal }}$ and $r_{\text {metal }}$ are the dielectric function and radius of the plasmonic NP, $R$ is the gap distance between the molecule and the plasmonic NP. $C_{\text {plasmon }}$ is proportional to $\operatorname{Im}\left(\varepsilon_{\text {metal }}\right)$, given that absorption at the plasmon resonance takes place in the metal NP. In more complex molecular-plasmonic systems, it becomes more difficult to address the analytical expression of the plasmon-induced CD term. However, after making reasonable approximations, it is possible to estimate $C D_{\text {plasmon }}$ as follows

$\mathrm{CD}_{\text {plasmon }} \propto \operatorname{Im}\left(\varepsilon_{\text {metal }}\right) \cdot f_{\text {resonant }} \cdot \operatorname{Im}\left[\left(\hat{K} \cdot \boldsymbol{\mu}_{12}\right) \cdot \boldsymbol{m}_{21}\right]$

where $\hat{K}$ is an electromagnetic matrix describing the interactions between the molecular dipole and a plasmon resonance, and $f_{\text {resonant }}$ is a hybrid function describing the frequency-dependent plasmonic enhancement. $\mathrm{CD}_{\text {plasmon }}$ will thus generate a CD signal at the plasmon resonance wavelength, which usually leads to a new peak in the CD spectrum (Figure 2B). ${ }^{15}$

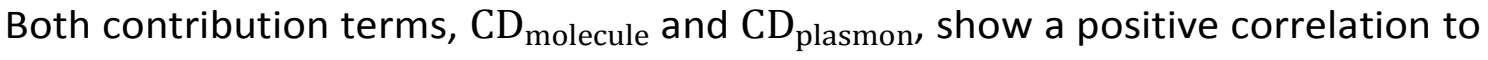
the plasmon-induced enhancement of the electric dipole. This enhancement effect is proportional to $\mathrm{R}^{-3}$, which means that it decreases rapidly as the molecule-NP distance increases. Therefore, obtaining an intense plasmonenhanced CD signal would require that the chiral molecule is located close to the surface of the plasmonic NP (Figure 2B). 
An alternative - though related - approach would involve placing the molecule within a plasmonic hotspot, i.e., a region of intense local field enhancement, usually at a small gap between two plasmonic nanostructuress. As an example, Figure $2 \mathrm{C}$ shows the case of a complex made of a nanoparticle dimer and a chiral molecule located at the gap between the NPs. ${ }^{17}$ Theoretical calculations and experimental observations have consistently shown that, the electromagnetic near field is much stronger at the interparticle gaps, thereby leading to larger enhancements of the $C D$ response. Such a hotspot-mediated plasmonic chirality can be artificially engineered, not only in intensity but also in position and handedness of the plasmonic CD peak. ${ }^{7}$

When the size of the plasmonic NP is comparable to $\lambda / 2$ of the incident light, the above-mentioned near-field induction model using the quasi-static approximation is no longer valid because of retardation effects. ${ }^{18}$ In general, the constitutive relations of the molecule-plasmonic system can be defined as

$$
\left\{\begin{array}{l}
\mathbf{D}=\varepsilon \mathbf{E}+\xi \mathbf{H} \\
\mathbf{B}=-\xi \mathbf{E}+\mu \mathbf{H}
\end{array}\right.
$$

where $D$ is the electric displacement field, $E$ and $H$ the electric and magnetic fields, $\mathrm{B}$ the magnetic induction and $\varepsilon, \xi$, and $\mu$ are parameters describing the properties of the chiral medium. $\varepsilon, \xi$, and $\mu$ are all $3 \times 3$ matrices due to the anisotropy of the chiral medium. ${ }^{9}$ Furthermore, this equation can be written in several ways as Tellegen's relations $^{19}\left\{\begin{array}{l}\mathbf{D}=\varepsilon_{0} \varepsilon_{\mathrm{r}} \mathbf{E}+i \kappa \sqrt{\mu_{0} \varepsilon_{0}} \mathbf{H} \\ \mathbf{B}=-i \kappa \sqrt{\mu_{0} \varepsilon_{0}} \mathbf{E}+\mu_{0} \mu_{\mathrm{r}} \mathbf{H}\end{array}\right.$

Post's relations $^{20} \quad\left\{\begin{array}{c}\mathbf{D}=\varepsilon_{\mathrm{p}} \mathbf{E}+i \xi_{c} \mathbf{B} \\ \mathbf{H}=i \xi_{c} \mathbf{E}+\mu_{\mathrm{p}}^{-1} \mathbf{B}\end{array}\right.$

Drude-Born-Fedorov $\left\{\begin{array}{l}\mathbf{D}=\varepsilon_{\mathrm{DBF}}(\mathbf{E}+i \chi \mathbf{B}) \\ \mathbf{B}=\mu_{\mathrm{DBF}}(\mathbf{H}-i \chi \mathbf{D})\end{array}\right.$

where $\varepsilon_{\mathrm{r}}, \varepsilon_{\mathrm{p}}$, and $\varepsilon_{\mathrm{DBF}}$ describe the permittivity of the medium, $\mu_{\mathrm{r}}, \mu_{\mathrm{p}}$, and $\mu_{\mathrm{DBF}}$ describe the permeability of the medium and $\kappa, \xi_{c}$, and $\chi$ describe the chiral properties of the medium. In the case of a nonmagnetic medium (i.e. $\mu_{\mathrm{p}}=1$ ), taking Post's relations as an example, the constitutive parameters $\left(\varepsilon_{\mathrm{p}}\right.$ and $\left.\xi_{c}\right)$ inside the formula can be derived as 
$\varepsilon_{\mathrm{p}}(\omega)=\varepsilon_{c}-\gamma_{c}\left(\frac{1}{\hbar \omega-\hbar \omega_{0}+i \Gamma_{12}}-\frac{1}{\hbar \omega+\hbar \omega_{0}+i \Gamma_{12}}\right)$

$\xi_{c}(\omega)=\beta_{c}\left(\frac{1}{\hbar \omega-\hbar \omega_{0}+i \Gamma_{12}}+\frac{1}{\hbar \omega+\hbar \omega_{0}+i \Gamma_{12}}\right)$

where $\varepsilon_{c}$ denotes the background refractive index, the coefficients $\gamma_{c}$ and $\beta_{c}$ determine the amplitudes of absorptive and chiral properties. The coupling between the plasmonic NP and the chiral molecule can be considered by a farfield electromagnetic induction model. In this case, the properties of the plasmoninduced CD signal modification are generally investigated in molecule-plasmonic NP systems where the plasmonic NP is coated with layers of chiral molecules or embedded inside a chiral medium.

Besides generating enhanced $C D$ via molecule-plasmon coupling, plasmonic nanostructures with an intrinsically chiral geometry can also provide strong CD signals. ${ }^{2}$ 6, 16, 18 As an example, Figure 2D shows right-handed twister and antitwister Au NPs exhibiting well-defined CD signals. ${ }^{16}$ The total CD signal can be calculated through the absorption, $\mathrm{Q}$, of the chiral twisted plasmonic nanostructure, which can be expressed as

$\mathrm{Q}_{\mathbf{k}}=\int \mathrm{d} V\langle\mathbf{j} \cdot \mathbf{E}\rangle_{t}=\operatorname{Im}\left(\varepsilon_{\text {metal }}\right) \frac{\omega}{2 \pi} \int \mathbf{E}_{\omega} \mathbf{E}_{\omega}^{*} \mathrm{~d} V$

$\mathbf{k}$ being the incident wavevector, $\langle\ldots\rangle_{\mathrm{t}}$ a standard time averaging and $\mathbf{j}$ the electrical current density. The integral represents the Joule heat generation in the whole volume of the plasmonic nanostructure. The directional CD for a certain wavevector $\mathbf{k}$ can then be defined as

$\mathrm{CD}_{\mathbf{k}}=\mathrm{Q}_{\mathbf{k},+}-\mathrm{Q}_{\mathbf{k},-}$

where $\mathrm{Q}_{\mathbf{k},+}$ and $\mathrm{Q}_{\mathbf{k},-}$ correspond to absorption for left- and right-handed circularly polarized light, respectively. Considering that plasmonic nanostructures are randomly oriented in a colloidal system, the total CD signal is the integral of all orientations over $\theta_{k}$ and $\varphi_{k}$, i.e. the incident angles of the electromagnetic wave. For small nanostructures, the expresion of the total $C D$ signal can be simplified by considering only the directional $C D$ at three perpendicular directions: 
$\mathrm{CD}=\left\langle\mathrm{CD}_{\mathbf{k}}\right\rangle_{\Omega_{\mathbf{k}}}=\frac{1}{4} \int \mathrm{CD}_{\mathbf{k}} \cdot \sin \theta_{k} \mathrm{~d} \theta_{k} \mathrm{~d} \varphi_{k} \approx$

$\frac{C D_{k \|+x}+C D_{k \|-x}+C D_{k \|+y}+C D_{k \|-y}+C D_{k \|+z}+C D_{k \|-z}}{6}=\frac{C D_{k \|+x}+C D_{k \|+y}+C D_{k \|+z}}{3}$

In the above paragraphs, we summarized the theory for the CD properties of a molecule-plasmonic system. However, an analytical solution of the CD signal can only be obtained for a few systems under strict conditions (e.g., single chiral molecule and small metal nanosphere). In most cases, it is impossible to calculate directly the CD signal. Nowadays, various numerical methods have been developed which enable the design and optimization of chiral plasmonic systems, such as discrete-dipole approximation (DDA), ${ }^{21}$ boundary element method (BEM), ${ }^{12}$ surface integral equation combined with method-of-moments (SIEMoM), ${ }^{11}$ finite-difference time domain (FDTD), and frequency-domain finiteelement methods (FEMs). ${ }^{22}$ These approaches have become popular, since they have been implemented in standard commercial software packages, such as COMSOL Multiphysics or Lumerical. ${ }^{19}$

The determination of the CD response for a certain chiral plasmonic system involves first the design of an appropriate model, which can be built by following different steps. First, the geometry of the plasmonic system is imported into the software, based on information obtained typically from electron microscopy analysis, and the electromagnetic fields are modified according to the selected constitutive relations. Second, the properties of the chiral medium (e.g. Eqs. 9 and 10) and plasmonic materials (e.g. using a Drude-Lorentz dispersion model) are directly written into the numerical model. Appropriate boundary conditions and mesh are then selected. ${ }^{16}$ Finally, the extinction responses of the model under different incident light (i.e., LCP and RCP) and the corresponding CD signals are calculated using a numerical method. ${ }^{23}$ The chiroptical activity of the plasmonic complex nanostructures mainly results from their intrinsic chiral permittivity or/and chiral structure, which exist in several cases: (i) chiral molecules adsorbed on the plasmonic NP surface; (ii) chiral array of plasmonic NPs built by chiral molecule/template; (iii) nanomaterials with a chiral shell or chiral twisted morphology. In summary, when plasmonic NPs meet with chiral 
molecules/templates, or display helical chiral structures, a significant chiroptical activity can be achieved.

\section{Synthesis of discrete chiral plasmonic NPs via wet chemical methods}

Recent progress in wet chemistry has allowed the development of different routes for the synthesis of chiral plasmonic NPs. Such synthetic routes can be classified into two main strategies: Metal-Molecule-Metal (3M) and 3D chiral NPs. Scheme 1 summarizes both strategies, which are based on seed-mediated methods since they comprise as a first step the synthesis of nanoparticle seeds with well-defined shape and crystallography, subsequently followed by growth on the preformed NP seeds, during which chirality is imparted. In 3M NPs (Scheme 1), the final nanostructure involves a metal-molecule-metal core-shell-shell configuration, in such a way that chiral molecules constitute the inner shell and/or are embedded in the outer metal shell. The coupling of chiral dipoles from the molecule with the electromagnetic near-field generated at the inner hot-spots will be responsible for the chiroptical response. On the other hand, 3D chiral NPs show a chiral morphology (are intrinsically dissymmetric), which is built up during the growth step, typically induced by chiral molecules present in the growth reaction medium.
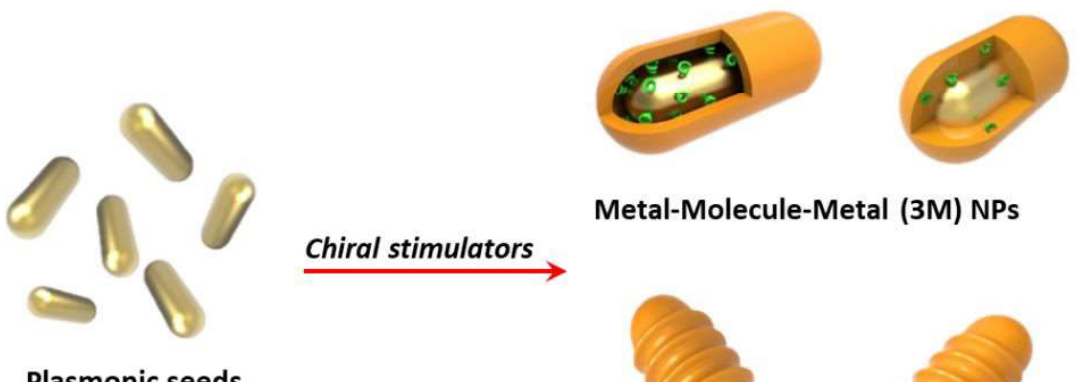

Metal-Molecule-Metal (3M) NPs

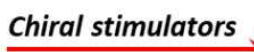

Plasmonic seeds

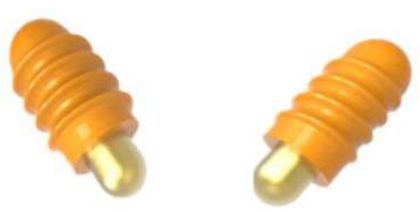

Chiral Plasmonic NPs

Scheme 1. Schematic representation of the two main strategies for the synthesis of chiral plasmonic nanoparticles, based on the seeded growth of pre-synthesised plasmonic seeds: Metal-Molecule-Metal (3M) NPs and Chiral Plasmonic NPs. 
Metal-Molecule-Metal (3M) nanoparticles. In what follows, we discuss the most relevant approaches so far, to synthesize discrete chiral plasmonic NPs. We commence with 3M NPs, which usually contain chiral molecules inside nanogaps or nanocavities present between a metal core and an outer metal shell. Such chiral molecules should thus contain suitable functional groups to promote binding onto the seeds surface and subsequent trapping during metal overgrowth. Different chiral biomolecules, such as peptides or DNA, which contain thiol and amine functional groups with strong binding affinity towards metal surfaces, have been used. The way chiral molecules adsorb onto the surface of the seeds, and therefore their surface distribution, are determined by parameters such as the crystalline structure and morphology of the metal seed, the concentration of chiral molecules, the bonding strength, etc.

Among the wide variety of available Au NPs, Au nanorods (NRs) are arguably the most commonly used morphology. The one-directional anisotropy of rod-like objects leads to a straightforward tunability of the so-called longitudinal LSPR, thereby offering interesting prospects. Single crystalline NRs have been studied in particular detail and can be readily synthesized within a wide range of sizes and aspect ratios, thereby being perfect candidates as seeds to explore the influence of chiral molecule concentration on the morphology and chiroptical properties of the resulting $3 \mathrm{M} \mathrm{NPs} .^{23}$ Zheng et al. investigated the influence of cysteine (Cys) concentration on the overgrowth of Au NRs (Figure $3 A$ )..$^{23}$ The results showed a conformal growth at low Cys concentrations (4 $\mathrm{nM}$ ), the appearance of one or two spikes at medium Cys concentration (40 $\mathrm{nM}$ ), and multiple spikes at high concentrations (400 nM). Such a Cys concentration-dependent growth was explained in terms of a surface blocking effect produced by the thiol group contained in the amino acid. Analysis of the chiroptical properties revealed that all overgrown Au NRs displayed bisignated plasmonic CD bands. Interestingly, those particles synthesized at medium Cys concentrations presented an anisotropy $g$-factor apparently related to the transverse LSPR of 0.021 , but also a plasmonic CD response associated with the longitudinal LSPR. Such a relatively high $g$-factor was attributed to efficient Cys encapsulation and intense hot spots 
generated at gaps between the Au core and the relatively thick outer Au shell, which enhanced the coupling of chiral dipoles with the electromagnetic near field. Moreover, the plasmonic CD bands of 3M Au NPs can be red-shifted by further coating them with silica, due to an increase in the dielectric permittivity of the sourrodunding medium (Figure $3 \mathrm{~A}$ ).
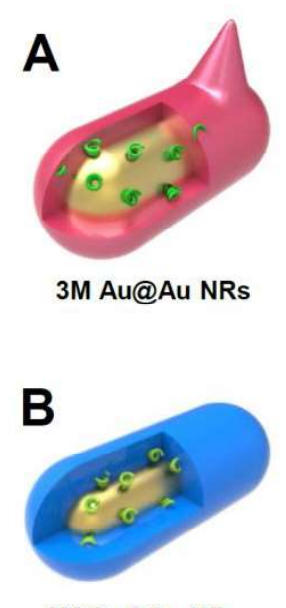

3M Au@Ag NRs

C

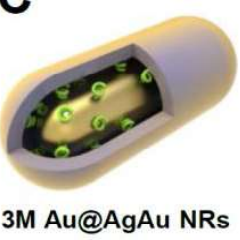

D

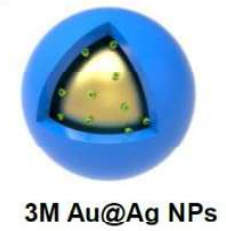

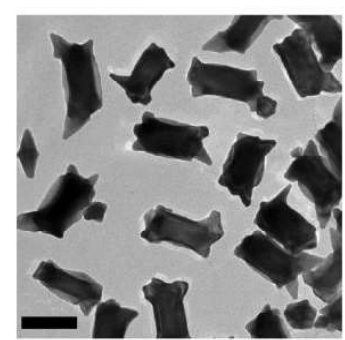
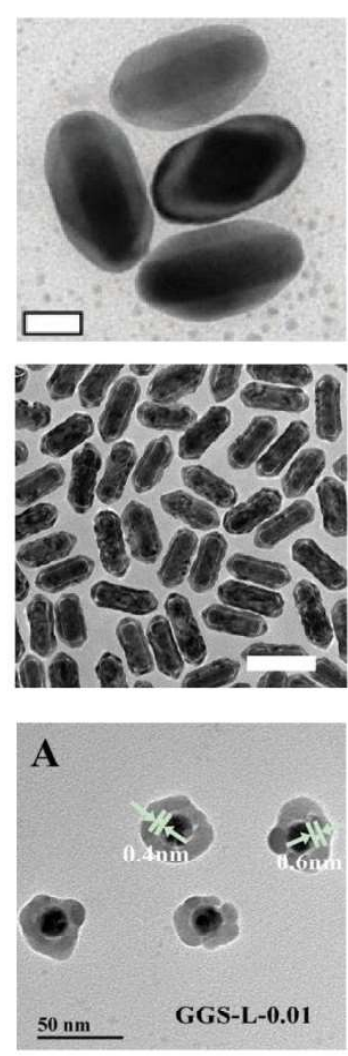
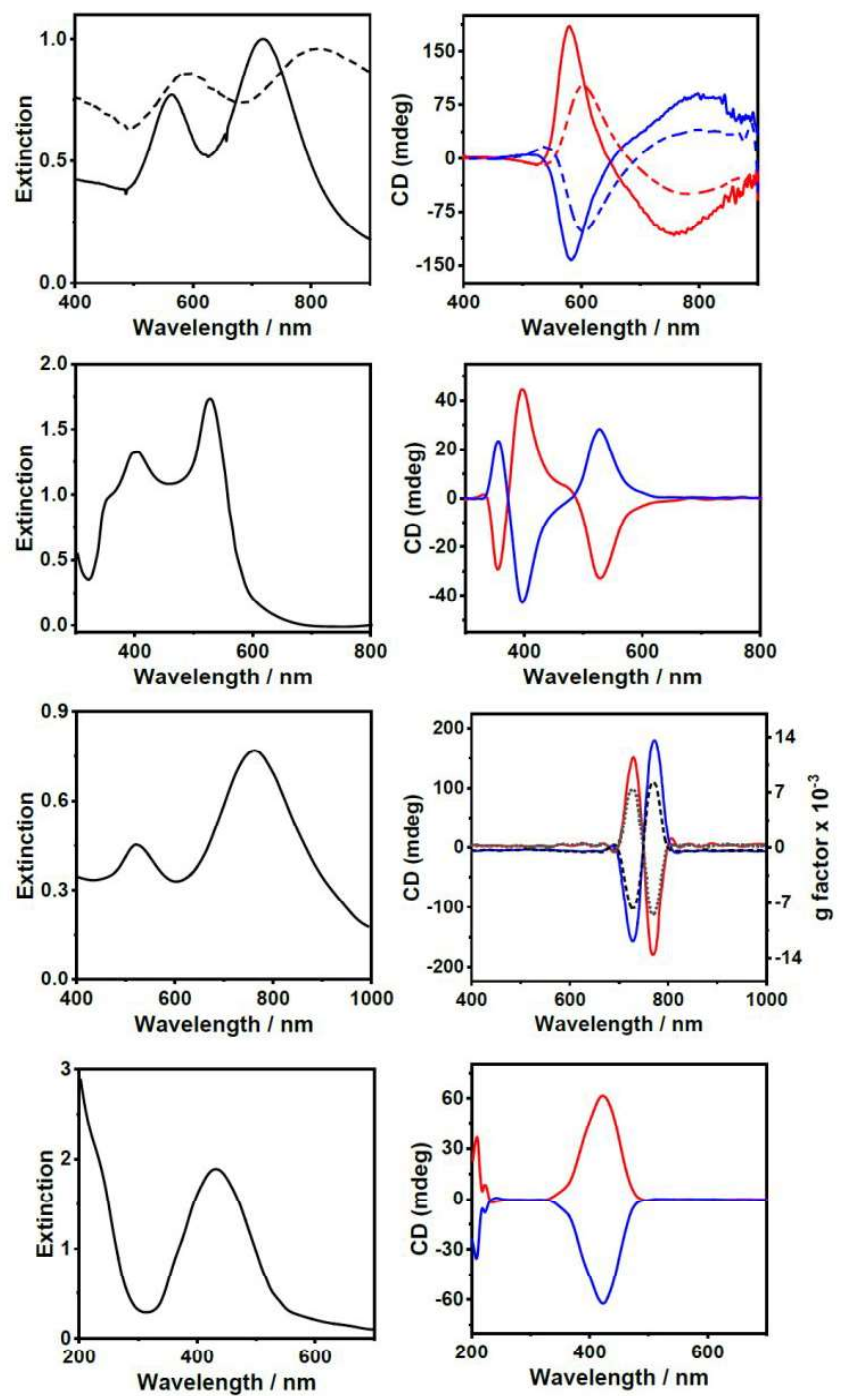

Figure 3. Schematic representation of different $3 M$ chiral NPs, together with representative TEM images and corresponding extinction and CD spectra. (A) Au@Au core-shell nanorods synthesized in the presence of L- or D-cysteine (Scale bar: $100 \mathrm{~nm}$ ). The dashed lines correspond to the same system after coating with silica, showing a red-shift in both extinction and CD spectra. Reproduced from ref. 23 Copyright 2018, Wiley. (B) Au@Ag core-shell nanorods synthesized in the presence of L- or D-cysteine (Scale bar: $20 \mathrm{~nm}$ ). Reproduced from ref. 24. 
Copyright 2016, Royal Society of Chemistry. (C) Au@AgAu yolk-shell nanorods synthesized in the presence of L- or D-penicillamine (Scale bar: $100 \mathrm{~nm}$ ). The dashed lines represent the estimated $g$-factors. Reproduced from ref. 25. Copyright 2018, Wiley. (D) Au@Ag core-shell spheres synthesized in the presence of cytosine-rich single-stranded DNA. Reproduced from ref. 26. Copyright 2015, Wiley.

Similar results were reported for both Cys and penicillamine-modified Au NRs, upon overgrowth with Ag (Figure 3B). ${ }^{24}$ Increasing the Ag shell thickness led to a blue-shift in both the LSPR and CD bands. The entrapment of amino acids within the $\mathrm{Au}-\mathrm{Ag}$ interface and the corresponding amplification of the electromagnetic near field were found to induce a moderate enhancement of the plasmonic CD response $(g$-factor $=0.0013)$. The chiroptical response of Au@Ag NRs was further improved by transforming the Ag shell into an AgAu yolk-shell NR structure via Galvanic replacement, which allowed to further engineer the interior nanogap and achieve a higher $g$-factor of 0.009 (Figure 3C). ${ }^{25}$ It should be noted that the Galvanic replacement process is accompanied by a red-shift in the optical and chiroptical properties of the particles.

A similar strategy has been demonstrated for core-shell nanospheres with interior nanogaps containing cysteine enantiomers, which confer them with plasmoninduced CD properties (Figure 3D). ${ }^{26,} 27$ The interior nanogap of Au@Ag spheres could be manipulated by varying Cys concentration, and anisotropy $g$-factors as high as 0.01 were reported. The plasmonic chiroptical properties are located at ca. $430 \mathrm{~nm}$ while in the UV region (200-250 nm) the characteristic CD peak of chiral molecules is observed. Alternatively, plasmonic CD signals were generated in Au@Ag nanospheres by using cytosine-rich single-stranded DNA as the template to guide silver shell growth. The DNA-plasmon interaction could be further amplified by electromagnetic coupling of the core-shell structure as the $\mathrm{Ag}$ shell thickness was increased, with a highest anisotropic $g$-factor of 0.0193 reported for a spherical NP. Unlike chiral NRs, the chiroptical response of spherical nanostructures does not offer a wide range of tunability. 
3D chiral nanoparticles. The construction of NPs with intrinsic three-dimensional chiral morphology (3D chiral NPs) is conceptually more challenging, since it involves drastic changes in the way metal atoms are deposited on the (achiral) seed NPs. As a result, the development of this approach has been slower and we can distinguish two main approaches among the few existing reports. A common concept between both strategies is the use of chiral molecules (such as peptides, aminoacids or surfactants) to dictate the seeded-growth on pre-formed NPs, confering them with chiral morphological features. Arguably, the most impressive results so far were obtained by Nam and co-workers, who reported the synthesis of so-called "plasmonic helicoids", with intricate twisted morphologies deriving from either cubic or octahedral NP seeds, using enantiomers of cysteine and glutathione as chirality inducers. ${ }^{28}$ Indeed, the origin of chirality in the final NPs was attributed to an enantioselective interaction between the chiral molecules and chiral atomic arrangements at the NP surface, such as high Miller index surfaces or kink sites. Chiral NPs were obtained by a seeded-growth process, typically using $50 \mathrm{~nm}$ cube-shaped gold seeds with low-index $\{100\}$ planes, which were overgrown in the presence of chiral amino acids, into NPs with high Miller index surfaces. The interaction of certain functional groups (thiol, amine, carboxylic, etc.) in the chiral molecules with the Au surface was proposed to play a critical role in controlling NP growth. Using this strategy, different helicoids with tailored handedness and strong chiral plasmonic resonances were fabricated.

It should be noted that, in the absence of chiral amino acids, the overgrowth of gold nanocubes resulted in the formation of symmetric and achiral stellated octahedra with 48 identical $\{321\}^{29}$ triangular facets in alternating $\mathrm{R}$ (clockwise) and S (anti-clockwise) conformation, which can be defined by the rotational direction of low-index planes $\{100\},\{110\}$, and $\{111\}$ (Figure 4). Oppositely, the presence of L-Cys or D-Cys enhanced the growth of right-handed or left-handed chiral nanostructures, as a result of different growth rates at the two oppositely chiral high-index planes of gold nanoparticles. Cysteine enantio-selectively interacts with $\{321\}$ high index surfaces at stellated octahedra, resulting in the splitting and growth of the boundaries between $\{321\}^{R}$ and $\{231\}^{S}$ facets of $R$ and $S$ regions in the [101] direction, causing the symmetry of the 
stellated octahedron to change from the $4 / \mathrm{m} 32 / \mathrm{m}$ point-group symmetry into 432 symmetry. Thus, when L-cysteine was used, R-S boundaries tilted by an angle - $\varnothing$ towards the $S$ region, whereas with $D$-cysteine it tilted towards the $R$ region by $+\varnothing$. This preferential growth was attributed to a different binding affinity of R and S kink sites with a specific cysteine enantiomer, hindering the vertical growth of that particular plane with respect to the other, and thereby causing the boundary to shift towards the plane with faster growth. Further, asymmetric growth along the [100] and [111] directions resulted in protruded vertices with split and twisted edges, creating a nmscale gap inside these helicoids (Figure 4C). These chiral NPs, referred to as 432 Helicoid I, featured a dissymmetry factor of 0.03 . By using glutathione molecules instead of cysteine in the growth solution, an overgrowth of the boundaries between $\{321\}^{R}$ and $\{312\}^{S}$ facets in the [011] direction was obtained. Although seeded growth occurred through the same intermediate nanostructure as that for 432 Helicoid I, the use of the larger glutathione molecule changed the interaction with the metal surface, giving rise to a different chiral nanostructure with pin wheel-like features and four-fold symmetry along the [100] direction (Figure 4D). These chiral NPs (432 Helicoid II) had a higher dissymmetry factor of 0.05 . Yet a different type of chiral nanostructure was obtained by using octahedral Au seeds enclosed by eight $\{111\}$ facets, in the presence of glutathione. The morphological evolution involved rhombic dodecahedral intermediates and eventually led to a pin wheel-like structure with more twisted features, and deeper gaps running radially from the center. A remarkable dissymmetry factor to 0.20 was recorded for these 432 Helicoid III NPs (Figure 4E), which represents a large increase with respect to 432 Helicoids I and II. In a subsequent improvement of the morphological homogeneity, the chiroptical response of these helicoid nanoparticles could be further enhaced up to 0.31 . Such an optimization involved a multi-chirality-evolution synthesis with precise control over the growth kinetics in a sequential growth process. ${ }^{30}$

The most recent studies from Nam's group focused on improving the chiral optical response of helicoid NPs. ${ }^{31}$ By carefully analysing the effect of various parameters, such as peptide sequence, seed morphology, or concentration of reactants, on the growth kinetics and the chiral features of the resulting NPs, both particles with improved chiroptical response and new helicoidal shapes could be obtained. 
A
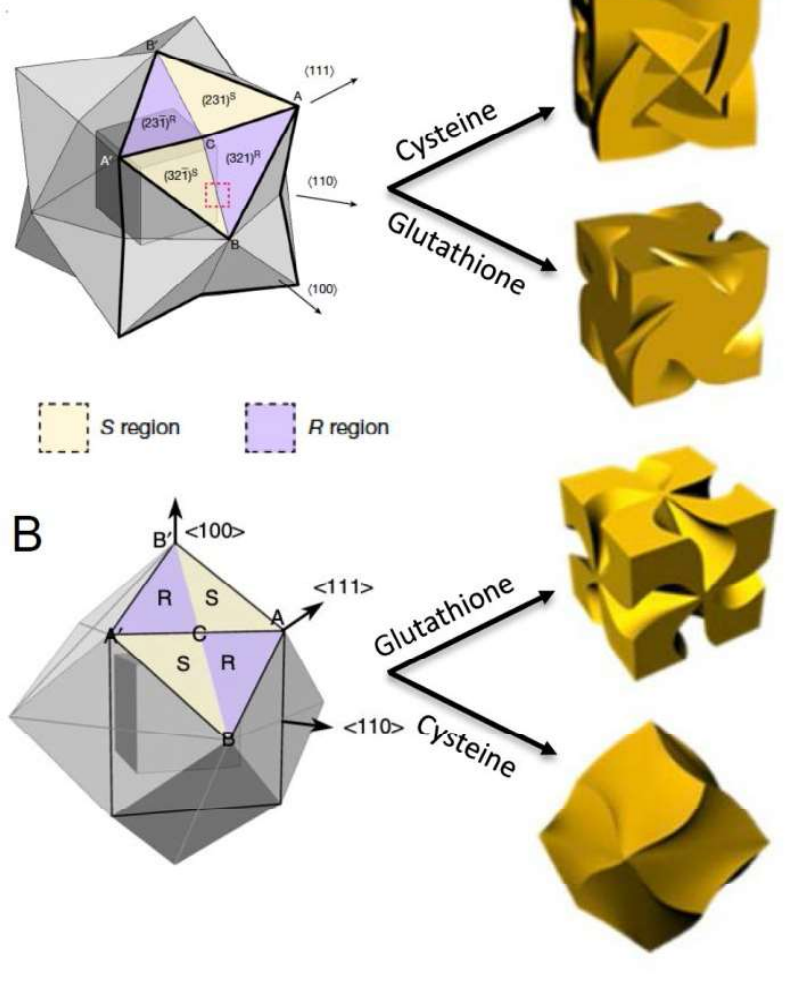
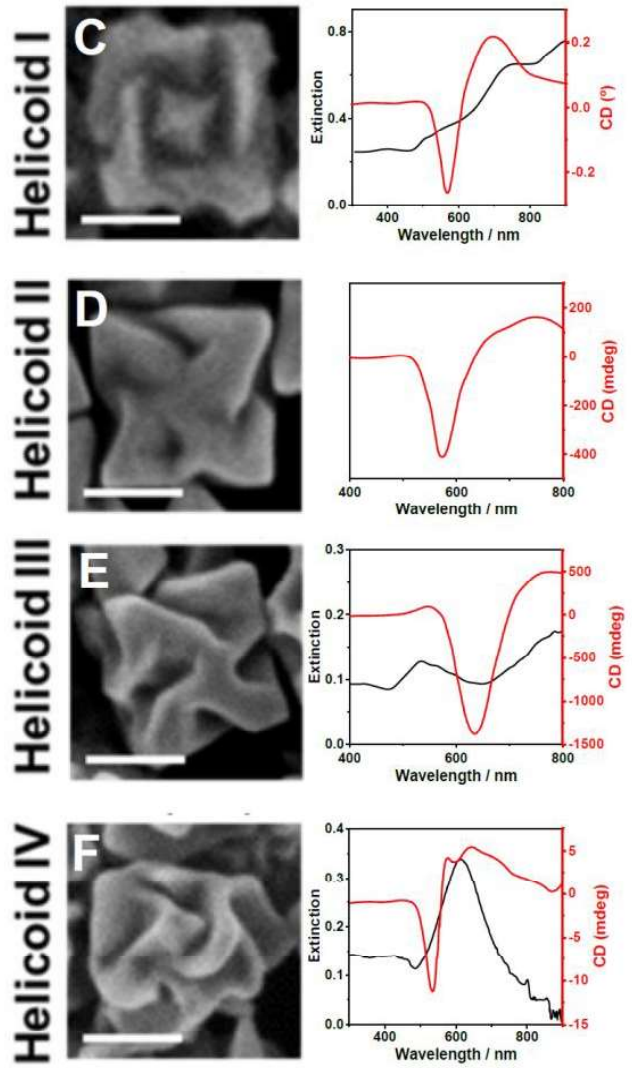

Figure 4. (A-B): Schematic representation of a stellated octahedron $(A)$ and rhombic dodecahedron (B), differentiated by high-index facets consisting of $S$ region and $\mathrm{R}$ region configurations, and the respective evolution of these nanostructures into different helicoids induced by the presence of cysteine and glutathione, as indicated. (C-F) Representattive SEM images and the corresponding UV-visible and CD spectra of: 432 Helicoid I (C), 432 Helicoid II (D), 432 Helicoid III (E), and 432 Helicoid IV (F). Adapted from refs. 28. Copyright 2018, Macmillan Publishers Ltd: Nature. Ref. 31. Copyright 2020, Macmillan Publishers Ltd: Nature Communications. Ref. 30. Copyright 2020, American Chemical Society.

A conceptually different approach, involving the use of chiral co-surfactants to template the growth of Au nanorods with screw-like helical features, was recently demonstrated by Liz-Marzan and co-workers. ${ }^{32}$ In this process, strong binding of chiral molecules was not required to direct dissymmetric growth. On the contrary, chiral co-surfactants such as 1,1'-bi(2-naphthol) (also known as BINOL) and 1,1'-binaphthyl-2,2'-diamine (BINAMINE) were used in combination with a quaternary ammonium surfactant (CTAC) to obtain chiroptically active, worm-like micelles, which adsorb on pre-formed Au NRs 
in a quasi-helical pattern, subsequently acting as templates to guide seeded-growth. As a result, pronounced wrinkles were obtained, which handedness was determined by the specific co-surfactant enantiomer used during growth. The chiral features on the overgrown NRs were significantly better defined when BINAMINE was used, which is likely related to the presence of two amine moieties in chelating configuration, with a higher affinity to the Au surface in comparison to the hydroxyl groups in BINOL. High quality electron tomography reconstructions of chiral nanocrystals obtained using (R)BINAMINE confirmed the presence of sharp wrinkles grown quasiradially, with tilt angles ranging from $0^{\circ}$ to $45^{\circ}$ with respect to the short AuNR axis, as well as an ill-defined structure when using (R)-BINOL (Figure 5A-D). A detailed analysis of orthoslices through the 3D reconstructions revealed an intricate network of wrinkles of varying heights, but with consistent width and inter-groove distances. Through 3D fast Fourier transformation, it was possible to identify the areas that contribute most to the chirality of the structure (Figure 5E). A correlation was observed, not only between the handedness of the helical structure with the enantiomeric form of the co-surfactant, but also between the size of micelles in solution and the measured interwrinkle distances on the NR surface. It was therefore suggested that the templating mechanism involves the micelles acting as patterns that direct the diffusion and surface-reduction of gold ions (likely complexed to $\mathrm{CTA}^{+}$molecules). The formation of sharp wrinkles is thus driven by a fast deposition rate of gold ions at available sites between CTAC/BINAMINE micelles on the NR surface. The extent of the wrinkles could be adjusted through the ratio between Au NR seeds and the reagents in the growth solution, leading to overall sizes ranging from $165 \times 73 \mathrm{~nm}$ to $270 \times 175 \mathrm{~nm}$. A most interesting observation was the possibility to tune the wavelength of plasmonic CD bands, from $700 \mathrm{~nm}$ up to $1600 \mathrm{~nm}$, by varying the dimensions of the wrinkles on Au NRs. As-synthesized chiral nanocrystals exhibited high dissymmetry factors in the visible ( $\sim 20)$, and estimated values close to 0.30 in the near IR (Figure $5 F, G$ ). The broad applicability of this method was demonstrated by overgrowing Pt grooves over Au NRs (Figure $5 \mathrm{H}-1$ ), as well as using spherical AuNPs as seeds (Figure 5J). 

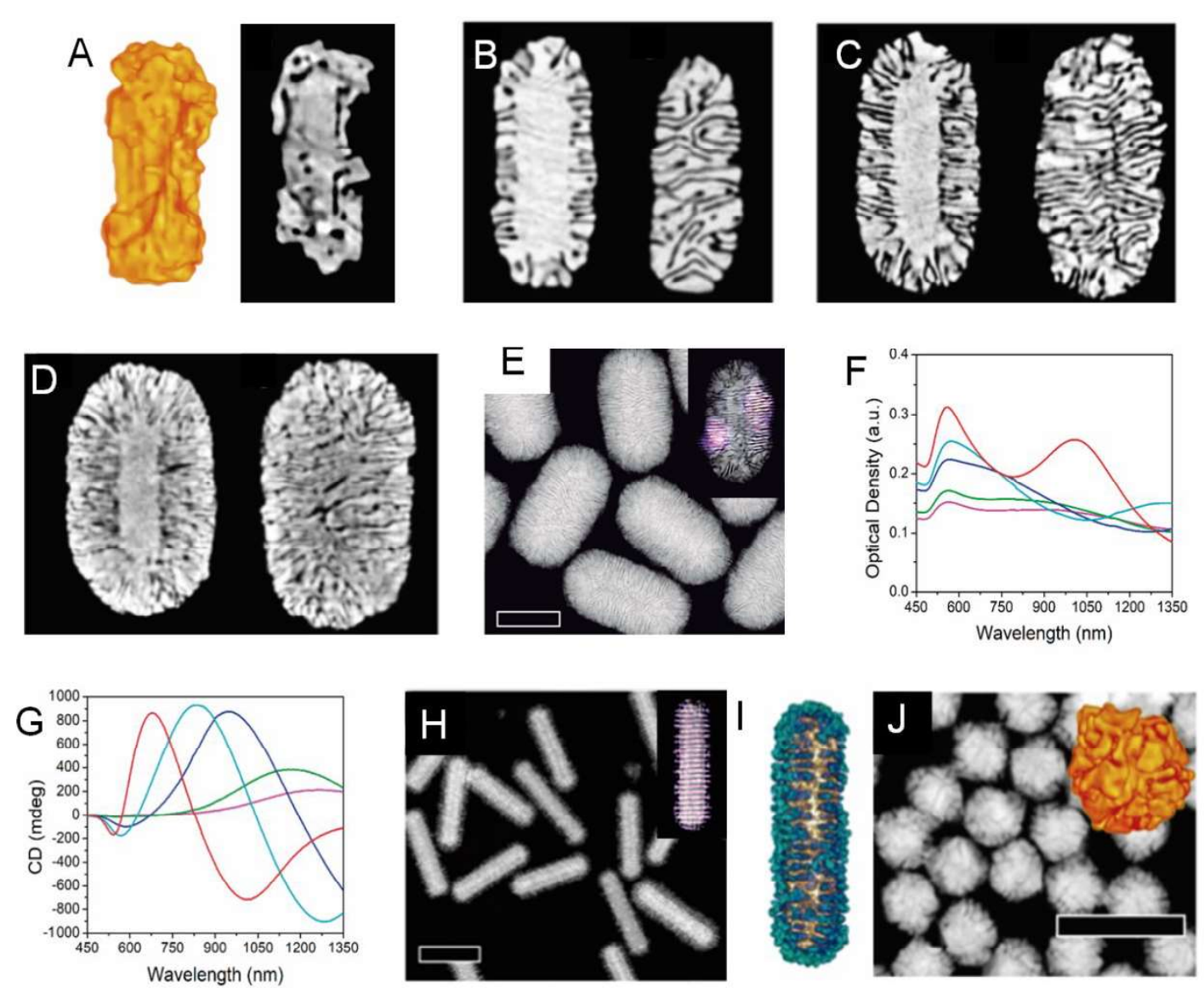

Figure 5. Chiral nanorods templated by helical surfactant micelles. (A) Tomography reconstruction of a gold nanorod overgrown in the presence of (R)-BINOL (left) and a selected orthoslice (right). (B-D) Orthoslices through 3D reconstructions from different chiral nanorods synthesized in the presence of (R)-BINAMINE, with sizes $165 \times 73 \mathrm{~nm}$ (B), $210 \times 112 \mathrm{~nm}$ (C), $270 \times 175 \mathrm{~nm}(\mathrm{D})$, revealing the presence of the central NR (left) and a radial wrinkle network (right). (E) HAADF-STEM Image of chiral 210x112nm nanorods (scale bar: $100 \mathrm{~nm}$ ) and an exemplary inverse FFT showing the areas of particle with chiral features, indicating right handedness (inset). (F,G) Vis-NIR (F) and CD (G) spectra of chiral nanorods with varying particle dimensions: 165x73nm (red), 200x92nm (cyan), 250x145nm (green), 270x175nm (magenta). (H) HAADF STEM Image of Au@Pt wrinkled nanorods (scale bar: $100 \mathrm{~nm}$ ) and inverse FFT (inset). (I) Tomography reconstruction of Au@Pt nanorods in EDX mode, showing the pattern of Pt grooves (cyan) on the central Au NR (golden); (J) HAADF-STEM Image of chiral nanospheres and a 3D reconstruction (inset). Reproduced from ref. 32. Copyright 2020, American Association for the Advancement of Science (AAAS).

Other approaches. Other plasmonic nanostructures with chiroptical properties have been reported, based on the use of soft and hard templates. For instance, 
Nakagawa and Kawai obtained double-helical Au nanowires with moderate optical activity, by using twisted nanoribbon soft templates obtained by mixing Dor L-12-hydroxystearic acid (HSA), which transferred the twisted conformation onto the nanowire helical nanostructures, and a long chain amidoamine (C18AA) serving as a capping agent for $\mathrm{Au}^{33}$ In another approach, inorganic nanostructures with chiral shapes were also proposed as hard templates to obtain chiral plasmonic nanostructures. Markovich and co-workers reported the synthesis of colloidal Te nanostructures with both chiral lattice and chiral shape resembling a trigonal prism with twisted ridges, ${ }^{34}$ using strongly binding chiral ligands and showing a large peak dissymmetry within the visible. Subsequently, chiral tellurium nanostructures were transformed into chiral gold nanostructures through galvanic replacement, resulting in a meaningful chiroptical activity $(g$ factor 0.003$)$. Another interesting approach made use of circularly polarized light as the source of chirality to be transferred onto the plasmonic nanostructures. Tatsuma and co-workers reported the fabrication of chiral plasmonic nanoparticles by selective deposition of a dielectric material such as $\mathrm{PbO}_{2}$ on the surface of achiral Au nanocuboids, taking advantage of plasmon-induced charge separation that occurs when the particle is in contact with a semiconductor. ${ }^{35}$ Depending on the handedness of the incident circularly polarized light, the localized electric field distributions around the achiral nanocuboids are twisted and localized at different corners, driven the selective deposition of $\mathrm{PbO}_{2}$ by $\mathrm{Pb}^{2+}$ oxidation. Furthermore, the handedness of the chiral plasmonic nanostructure could be reversibly switched between right and left by a reduction-oxidation process of $\mathrm{PbO}_{2}$ under UV and circularly polarized light, respectively. ${ }^{36}$

\section{Applications of discrete chiral plasmonic NPs}

We discuss in this section promising applications for discrete chiral NPs, with a primary focus in the fields of chemical sensing, circularly polarized photocatalysis, and photodynamic therapy. Although the emphasis will still be on chemically grown NPs, we also consider some applications that were initially developed using chiral NPs fabricated through physical methods, on the basis of their exceptional chiroptical performance. 
Chemical sensing. Label-free LSPR sensors rely on the high sensitivity of plasmonic nanostructures toward changes in the refractive index of their local surrounding medium. Typically, any change in the nanostructures environment, for instance related to molecular binding, can be readily tracked by an LSPR shift. ${ }^{37}$ LSPR-based sensors have demonstrated a relatively high sensitivity and speed, as well as simple-to-operate detection, and are therefore considered as efficient chemical sensors, even though they usually feature modest figures of merit (FOMs). Chiral plasmonic nanoparticles offer an attractive alternative, since they are characterized by rich CD spectral features with bipolar nature and multiple zero-crossing points, thereby maximising LSPR sensitivity and FOM (Figure 6A-D). Jeong et al. developed an analytical model for chiral plasmonic sensing that clearly shows the relevance of chirality and material properties in the sensing performance. ${ }^{38}$ Considering that the FOM is defined as the relationship between refractive index sensitivity and accuracy (standardised as the full width at half maximum, FWHM), the following expression can be derived:

$$
\mathrm{FOM}=2 \bar{\chi}|\delta \chi| \mathrm{n}^{3} / \varepsilon_{i}^{2} \sigma
$$

Where $\bar{\chi}$ is an achiral term, $\delta \chi$ is a chiral shape factor specific to right or left handed enantiomers, $\mathrm{n}$ is the refractive index of the local medium, $\varepsilon_{i}$ is the imaginary part of the dielectric constant and $\sigma$ the instrument resolution.

This equation clearly indicates that the chiral shape factor, $\delta \chi$, plays a decisive role in the FOM, so that an increase in $\delta \chi$ gives rise to a larger FOM. Considering this rule, the same authors used a physical vapour deposition technique to fabricate alloyed Ag-Ti nanohelices where the chiroptical properties could be tuned by adjusting the chiral shape factor and the dielectric function of the nanoparticles, by varying the $\mathrm{Ag}$ and $\mathrm{Ti}$ content. Interestingly, the sensitivity of the obtained nanohelices could be improved by increasing either their length (which increases the chiral shape factor), or the percentage of $\mathrm{Ti}$ in the alloy (flattering the dielectric function). As a result, these hybrid nanohelices could exhibit sensitivities of $\sim 1100 \mathrm{~nm}$ per refractive index unit (RIU) with a FOM of $\sim 2900 \mathrm{RIU}^{-1}$, which are large values considering those previously reported for 
LSPR sensors. ${ }^{38}$ As a proof of concept, the specific detection of biotin-avidin interaction was demonstrated.

Chiral nanostructures based on the assembly of chiral plasmonic nanoparticles have been demonstrated to perform particularly well in chemical sensing. The key concept is that the interparticle coupling (or hybridization) of chiral plasmon modes results in an enhanced chiroptical activity and a correspondingly emhanced sensitivity. For instance, Xu et al. proposed 3M Au@AgAu chiral NRs and Au nanospheres, as building blocks for chiral core-satellite assemblies mediated by DNA hybridization of complementary strands on each component. ${ }^{25}$ These chiral nanostructures display much stronger plasmonic $C D$ intensity and larger $g$-factors than the individual building blocks (0.021 vs 0.009, respectively), likely due to cooperative chirality resulting from combination of the structural chirality of the nanorods and plasmon-induced molecular chirality. Interestingly, chiral plasmonic core-satellite nanostructures fabricated using a $\mathrm{Zn}^{2+}$-specific DNA-cleaving DNAzyme were applied to quantitatively detect zinc ions in living cell lines (primary uterine fibroblast cells and HeLa cells). In the presence of the target $\mathrm{Zn}^{2+}$, the DNAzyme strand catalyses the cleavage of the complementary DNA strand on the Au satellites, thereby inducing disassembly of the core-satellite structure. By monitoring the resulting decrease in $C D$ response, $\mathrm{Zn}^{2+}$ concentration could be quantitatively determined (Figure 6E and F). 

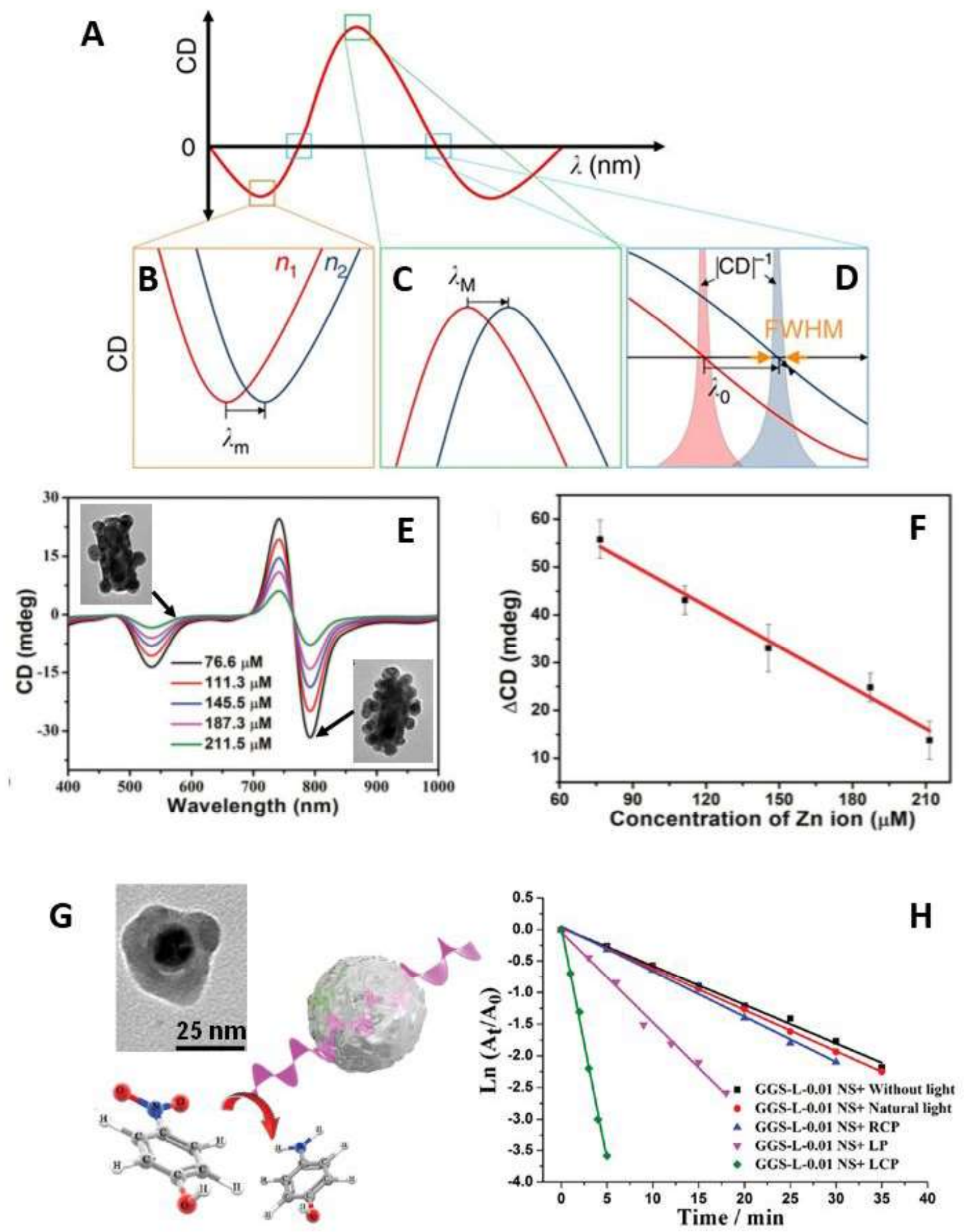

Figure 6. (A-D) Chiral plasmonic sensing. (A) CD spectrum of a plasmonic enantiomer as a function of wavelength. (B-D) Resonance shifts at the minimum $\lambda_{m}(B)$, maximum $\lambda_{M}$ (C) and half maximum $\lambda_{0}(D)$, where the refractive indices of the surrounding media are varied from $n_{1}$ to $n_{2}$. Reproduced with permission from ref. 38. Copyright 2015 Nature publishing group. (E-F) CD spectral changes with different concentrations of intracellular $\mathrm{Zn}^{2+}(\mathrm{E})$ and calibration curves for $\mathrm{Zn}^{2+}$ detection $(\mathrm{F})$, where $\triangle \mathrm{CD}=\mathrm{CD}_{740 \mathrm{~nm}}-\mathrm{CD}_{795 \mathrm{~nm}}$. The insets in (E) are representative TEM images showing a decrease in the number of $A u$ 
satellites in the assembly with increasing concentration of $\mathrm{Zn}^{2+}$ ions. Reproduced with permission from ref. 25. Copyright 2018, Wiley. (G-H) Schematic representation of the circularly polarized photocatalysis of 4-nitrophenol to 4-aminophenol by 3M Au@Ag chiral NPs (G). The inset shows a representative TEM image of a 3M Au@Ag chiral NP. (H) Kinetic trace (plot of $\operatorname{Ln}\left(A_{t} / A_{0}\right)$ as a function of time) for the reaction catalyzed by $3 M$ Au@Ag chiral NPs under different light conditions (RC, L and LC stand for right-circularly, linear and left-circularly polarized light, respectively). Reproduced with permission from ref. 27. Copyright 2015, Wiley.

Enantioselective chemical sensing is a field in which chiral plasmonic nanoparticles may play a particularly important role. Liu et al. recently proposed a chiral nanostructure comprising a gold nanorod coated with a chiral mesoporous silica shell to semiquantitatively measure the composition of chiral enantiomers by means of SERS. ${ }^{39}$ The growth of the chiral shell onto the Au NRs was directed by using amino acid-derived chiral surfactants as the template molecules. The strong near-field coupling between Au NR and chiral molecules on the shell confers the system with a strong chiroptical activity in the visible and NIR regions. By simply adding chiral cysteine molecules, they get infiltrated in the porous shell, as demonstrated by SERS spectroscopy. Interestingly, when cysteine molecules and the shell present the same handedness a new peak appears in the SERS spectrum, but this was not evident with cysteine and a shell of opposite chirality. The difference in SERS spectra was ascribed - and demonstrated by theoretical calculations - to a conformational change of chiral cysteine molecules when they are docked inside chiral mesoporous silica shells with the same handedness. Additionally, a semi-quantitative determination of the amount of cysteine enantiomers in solution was demonstrated, thereby becoming a promising platform for the sensitive SERS determination of enantiomeric ratios.

Circularly polarized photocatalysis. Plasmonic nanostructures exhibiting large chiroptical asymmetries are expected to generate plasmon-induced hot electrons, which are intimately related to near-field enhancement, 40 and therefore dependent on the polarization of incident circularly polarized light. 
Fang and co-workers demonstrated dichroic hot electron transfer from chiral Au crescent-like nanoparticles to $\mathrm{TiO}_{2}$, where differences in the incident photon-to-charge conversion efficiency (IPCE) between right- and left-handed circularly polarized illumination were in agreement with standard CD extinction measurements. ${ }^{41}$ Additionally, by using cathodoluminescence spectroscopy they were able to show that the chiral response is caused by the polarization dependence of the local field hot-spot distributions associated with the corresponding plasmon resonances. As a result, the circularly dichroic effect of asymmetric hot electrons could enhance different photochemical processes. On a related report, Xu et al. studied the effect of circularly polarized light on the catalytic performance of 3M Au@Ag chiral NPs towards the reduction of 4-nitrophenol (4-NP) into 4-aminophenol (4-AP), in the presence of borohydride. This reaction, which takes place at the surface of metal nanoparticles, is a well-known model to evaluate the catalytic activity of nanocatalysts and can be readily monitored by UV-visible spectroscopy. ${ }^{42}$ The results showed that, irradiation with circularly polarized light with the same handedness as the plasmonic nanoparticles gave rise to catalytic efficiencies at least 10 -fold higher than the photocatalytic reaction with linearly polarized, with natural light or in the absence of light (Figure 6G and 6H). ${ }^{27}$ Such a catalytic performance was ascribed to the increased generation of hot electrons by chiral plasmonic nanoparticles when irradiated with light of matching circular polarization.

Circularly polarized photodynamic therapy. Photodynamic therapy combines light energy with a drug (photosensitizer), which is initially non-toxic but is designed to induce cell death (by reactive oxygen species (ROS) generation) upon activation by light. Plasmonic nanoparticles can readily display higher extinction coefficients than standard organic photosensitizers and can therefore be used to transfer energy to molecular oxygen with high efficiency and selectivity for the generation of singlet oxygen species $\left({ }^{1} \mathrm{O}_{2}\right)$. The efficacy strongly relies on the optical properties of plasmonic nanoparticles, since plasmons can delay the recombination rate of electrons and holes. ${ }^{43}$ Therefore, chiral plasmonic nanoparticles should be able to act as photosensitizers under circularly polarized light irradiation. For instance, Gao et al. proposed DNA-driven core-satellite Au 
assemblies as chiral photosensitizers. ${ }^{44}$ These nanostructures, exhibiting chiroptical properties in the visible region, show highly efficient ROS generation at least three times higher than that provided by protoporphyrin IX, a standard organic photosensitizer. Illumination with either circularly polarized light with opposite handedness or linearly polarized light, did not induced ROS production, which was ascribed to insufficient energy transfer efficiency. The potential of such core-satellite assemblies as chiral photodynamic therapy agents was demonstrated both in vitro and in vivo.

Chiral Photothermal Effects. Upon LSPR excitation of a plasmonic nanoparticle with light, enhanced absorption and/or scattering processes occur at the NP surface. Both processes depend on the nature and the shape of the particles, absorption scales linearly with volume, whereas scattering varies as volume squared. Plasmonic nanostructures can thus convert absorbed light into heat, and subsequently transfer this energy to the surrounding medium giving rise to a temperature increase. ${ }^{45}$ This thermal dissipation (or lattice cooling) is responsible for well-known photothermal effects, which have been used for biomedical applications. Similarly, photothermal chirality originating from the differential absorption of circularly-polarized light by chiral plasmonic nanoparticles could also be exploited in various fields. Miandashti and coworkers experimentally demonstrated photothermal chirality originating from the differential absorption of circularly-polarized light by left-handed Au helicoids. ${ }^{46}$ The photothermal response was measured by luminescence thermometry through spin-coating the chiral nanoparticles on a AlGaN: $\mathrm{Er}^{3+}$ thin film. Upon LSPR excitation, an increase in the temperature, attributed to the absorption cross section of the Au helicoids, was observed. A comparative study, performed between LCP and RCP illumination of spin-coated Au helicoids, showed a net increase in temperature of $6 \mathrm{~K}$ when irradiated with RCP compared to LCP illumination. Additionally, a 12fold increase of the laser excitation intensity generated a temperature increase as high as $50 \mathrm{~K}$. Interestingly, since the temperature increment is directly proportional to both the absorption cross section and the laser intensity, optical and photothermal effects should produce similar $g$-factors, i.e. if a chiroptical $g$ - 
factor of 0.1 were observed, a 10\% higher temperature would be obtained for RCP light illumination.

Other applications. $C D$ is mainly caused by molecular electron energy level transitions, due to differences in the refractive index and absorption coefficient of optical materials. Therefore, the characterization of enantiomers is usually carried out by measuring CD spectra, based on the extinction response to RCP or LCP light. Additional information may be obtained by analysing the vibrational modes under irradiation with RCP or LCP light. Thus, vibrational CD (VCD) and Raman optical activity (ROA) spectra are associated with molecular vibrational modes and carried out with near-infrared laser irradiation. Bearing this concept in mind, chirality transfer from one molecule/nanostructure to another could also be of relevance toward the development of new applications in chiral technology. Dolamic et al. showed that, an intrinsically chiral gold cluster can transfer its handedness to an achiral molecule, such as 2-phenylethylthiolate, adsorbed on its surface. ${ }^{47}$ VCD spectra showed certain signals associated with the preferential adsorption of the molecules adopting a chiral conformation. In a different approach, Pour and coworkers demonstrated the chirality transfer from a chiral molecule (ribose or tryptophan) into an achiral plasmonic nanostructure comprising $\mathrm{Ag} @ \mathrm{SiO}_{2}$ core-shell nanoparticles doped with a resonant Raman achiral dye (benzotriazole, with an absorption maximum at $514 \mathrm{~nm}$ ). Covalent surface modification of $\mathrm{Ag} @ \mathrm{SiO}_{2}$ NPs with the chiral molecule induced a symmetry breaking of the LSPR, which became chiroptically active as demonstrated by the opposite mirror bands exhibited in the ROA spectra recorded for both enantiomers of the chiral molecule (Figure 7A). This is the first report of chirality transfer from a chiral molecule to an achiral one, mediated by a plasmonic nanoparticle. This approach could open a new route to study the conformation of molecules near a plasmonic surface for enantiomer recognition. Plasmonic nanostructures with large $g$-factors can selectively absorb circularly polarized light with the same handedness while transmitting the other. This phenomenon could be applied to the design of broadband circular polarizers or for colour modulation. In the first case, Gansel et al. proposed a photonic 
metamaterial consisting of chiral plasmonic Au helices with mid-IR absorption, arranged on a two-dimensional square lattice as a compact broadband circular polarizer. ${ }^{48}$ By tuning the number of helix pitches they demonstrated experimentally that the transmittance of circularly polarized light with the same handedness was very low (below 5\%) in a broad band range (from 3.75 to $7.5 \mu \mathrm{m}$, see Figure 7B). In the latter case, Lee et al. reported the use of chiral helicoid NPs with high anisotropy factor for circular polarization-dependent transmitted colours under cross-polarized conditions. ${ }^{28}$ Figure 7C shows the polarizationresolved colours of light transmitted through dispersions of helicoid chiral particles with LSPR peaks ranging from $552 \mathrm{~nm}$ to $668 \mathrm{~nm}$, at different rotational angles between crossed polarizers. Interestingly, the colour transformation of the different colloids was continuous and asymmetric with angle, thereby covering a wide colour range. 

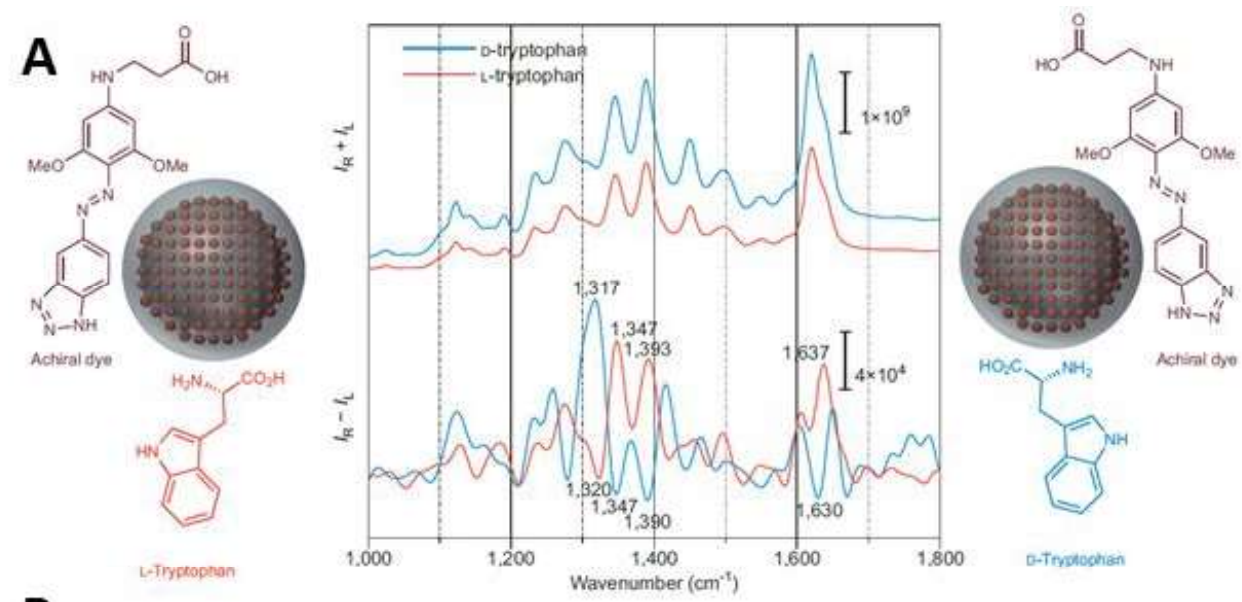

B
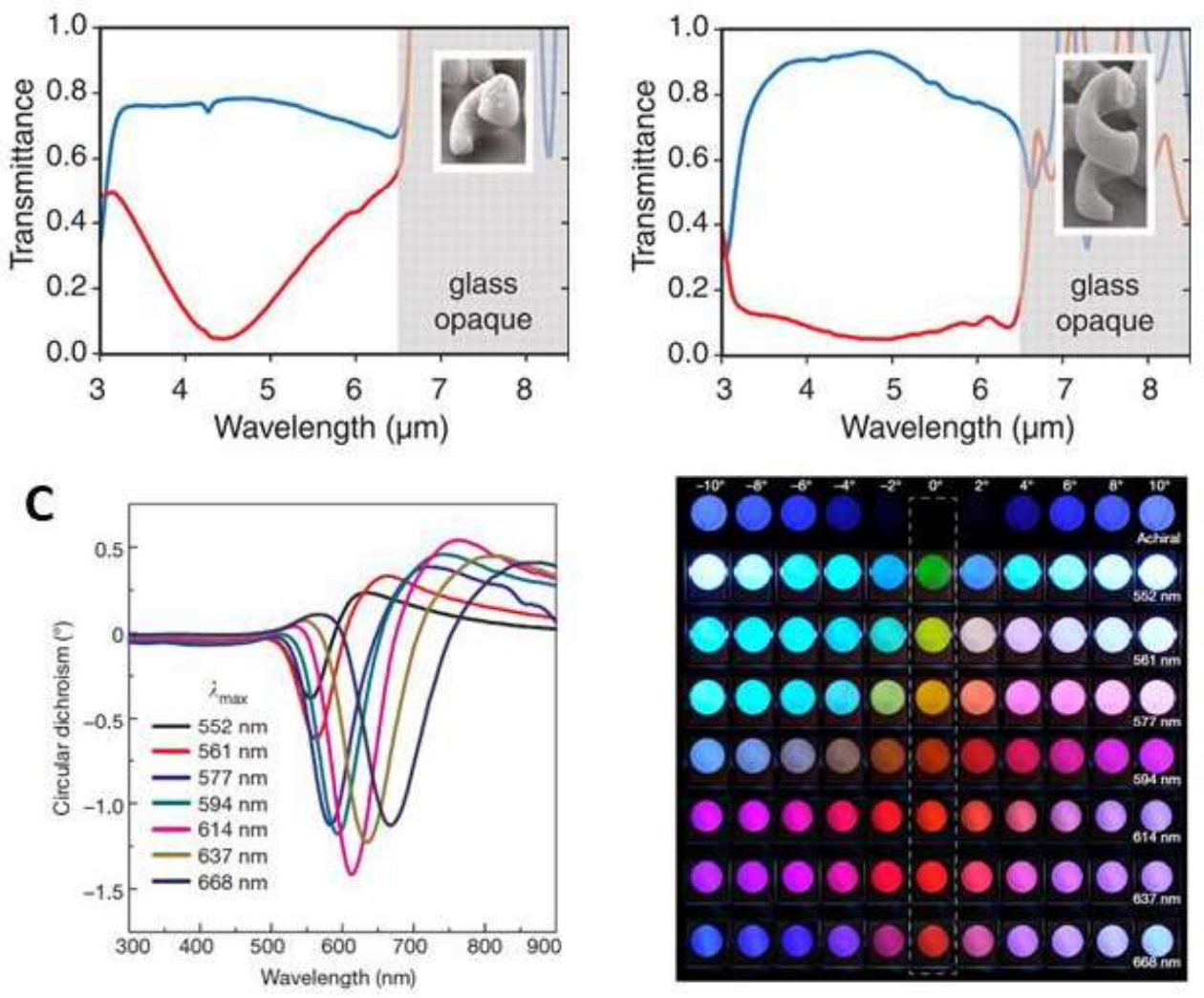

Figure 7. (A) Chirality transfer from a chiral analyte to an achiral reporter. The SERRS (resonant SERS) spectra (top) of D- and L-tryptophan bound to benzotriazolefunctionalized nanotags are identical - as expected for two enantiomeric systems. Surface-enhanced resonant Raman optical activity (SERROA) spectra (bottom) for both systems show strong chiroptical responses that are attributed to the achiral dye. $I_{R}$ and $I_{L}$ are the intensities of right- and left-polarized light, respectively. Reproduced with permission from ref. 49. Copyright 2015 Nature publishing group. (B) Transmittance spectra measured through a photonic metamaterial composed of a $2 \mathrm{D}$ square lattice of slightly less than one pitch of left-handed helices (left) and two pitches of left-handed helices (right). Red and blue spectra correspond to the illumination with right- and left- 
handed circular polarization, respectively. Reproduced with permission from ref. 48. Copyright 2009 American Association for the Advancement of Science (AAAS). (C) (Left) CD spectra of Au helicoid NPs for different values of $\lambda_{\max }$ (the wavelength of maximum $g$ factor) and (Right) polarization-resolved colours of light transmitted through seven different $\mathrm{Au}$ helicoid NPs dispersions with different $\lambda_{\max }$ values (shown in the corresponding CD spectra) and an achiral nanoparticle solution (top row). Reproduced with permission from ref 28. Copyright 2018 Nature publishing group.

\section{Conclusions and outlook}

The various examples discussed above allow us to foresee that wet-chemical approaches have a great potential toward the synthesis of discrete metal nanoparticles with strong and tuneable plasmonic chirality. Notwithstanding, much remains to be accomplished in this field, encompassing from improved synthesis to reliable theoretical models and real applications. In the following, we highlight several research directions that appear particularly important to be fulfilled in the near future.

The positioning of chiral molecules close to, in contact, or embedded in an achiral plasmonic nanoparticle lead to plasmon-induced chirality and CD response at the LSPR wavelengths, typically in the visible and NIR range. Nevertheless, a more comprehensive theoretical understanding is needed to correlate the positioning and orientation of the chiral molecules on the nanoparticle with their chiroptical activity. Another parameter that has not been studied in sufficient detail but can strongly affect the chiroptical activity of discrete metal nanoparticles, is the nature of the chiral molecule and how it couples to plasmon-derived fields. Other parameters producing dichroism in the vis-NIR plasmonic regime, apart from the absorption-mediated chiral response of the molecules, e.g. the molecule and NP refractive indices, should be explored. Most studies are still focused on $\mathrm{Au}$, but other plasmonic metals and dielectric materials, including silver, aluminium or palladium, will be worth exploring.

The development of novel synthetic approaches to obtain discrete chiral nanoparticles with high morphological and chemical stability, as well as intense plasmonic chirality, can expand their applicability in different technologies. We envision widespread use in 
the fabrication of chiral optical devices, enantioselective catalysis, chiral selective sensing, and chirality separation filters, among others.

Regarding the synthesis of 3D chiral nanoparticles, better control over their shape and size is still needed to achieve tunable chiroptical responses over a broader spectral range. Furthermore, understanding the origin of chirality in such colloidal particles is essential to further explore efficient synthesis approaches. The relationship between the extinction spectrum of 3D chiral nanoparticles and their chiroptical activity should be studied in more detail.

Another topic that will surely deserve additional attention is the directed assembly of chiral plasmonic nanoparticles and the implications of this process in the generation of chiral responses based on plasmon coupling. In this context, the development of stimuliresponsive self-assembled NPs with plasmonic chirality deserves particular attention. ${ }^{50}$ DNA nanotechnology and other stimuli-responsive self-assembly approaches will definitely be applied to synthesize chiral plasmonic nanostructures for further applicability in sensing, catalysis or information storage.

\section{Conflicts of interest}

There are no conflicts to declare.

\section{Acknowledgements}

G. Z. acknowledges the National Natural Science Foundation of China (Grant Number: 21902148). Financial support was also provided by the Spanish Ministerio de Ciencia e Innovación (Grant No. PID2019-108954R to I. P.-S. and J. P.-J.; Grants No. MAT201786659-R and MDM-2017-0720 to L. M. L.-M.). G. Z. and K.-Y. W. acknowledge the financial support from the Innovation and Technology Commission of Hong Kong and The Hong Kong Polytechnic University.

\section{References}

1 A. Cecconello, L. V. Besteiro, A. O. Govorov and I. Willner, Nat. Rev. Mater., 2017, 2, 17039.

2 J. Kumar, H. Erana, E. Lopez-Martinez, N. Claes, V. F. Martin, D. M. Solis, S. Bals, A. L. Cortajarena, J. Castilla and L. M. Liz-Marzan, Proc. Natl. Acad. Sci. U.S.A., 2018, 115, 3225-3230. 

Zinic, M. M. Cid and L. M. Liz-Marzan, Angew. Chem. Int. Ed., 2011, 50, 5499-5503. X. L. Wu, C. L. Hao, J. Kumar, H. Kuang, N. A. Kotov, L. M. Liz-Marzan and C. L. Xu, Chem. Soc. Rev., 2018, 47, 4677-4696.

5 A. G. Mark, J. G. Gibbs, T. C. Lee and P. Fischer, Nat. Mater., 2013, 12, 802-807.

6 A. Kuzyk, R. Schreiber, Z. Y. Fan, G. Pardatscher, E. M. Roller, A. Hogele, F. C. Simmel, A. O. Govorov and T. Liedl, Nature, 2012, 483, 311-314. B. Han, Z. N. Zhu, Z. T. Li, W. Zhang and Z. Y. Tang, J. Am. Chem. Soc., 2014, 136, 1610416107.

8 M. J. Urban, C. Q. Shen, X. T. Kong, C. G. Zhu, A. O. Govorov, Q. B. Wang, M. Hentschel and N. Liu, Annu. Rev. Phys. Chem., 2019, 70, 275-299.

J. T. Collins, C. Kuppe, D. C. Hooper, C. Sibilia, M. Centini and V. K. Valev, Adv. Opt. Mater., 2017, 5, 1700182.

10 C. Zhou, X. Y. Duan and N. Liu, Acc. Chem. Res., 2017, 50, 2906-2914.

11 D. M. Solis, J. M. Taboada, F. Obelleiro, L. M. Liz-Marzan and F. J. G. de Abajo, ACS Nano, 2014, 8, 7559-7570.

12 V. Myroshnychenko, J. Rodriguez-Fernandez, I. Pastoriza-Santos, A. M. Funston, C. Novo, P. Mulvaney, L. M. Liz-Marzan and F. J. G. de Abajo, Chem. Soc. Rev., 2008, 37, 1792-1805.

13 X. T. Kong, L. V. Besteiro, Z. Wang and A. O. Govorov, Adv. Mater., 2018, e1801790.

14 A. O. Govorov, Z. Y. Fan, P. Hernandez, J. M. Slocik and R. R. Naik, Nano Lett., 2010, 10, 1374-1382.

15 A. O. Govorov, J. Phys. Chem. C, 2011, 115, 7914-7923.

16 Z. Y. Fan and A. O. Govorov, Nano Lett., 2012, 12, 3283-3289.

17 H. Zhang and A. O. Govorov, Phys. Rev. B, 2013, 87, 075410.

18 B. Auguie, J. L. Alonso-Gomez, A. Guerrero-Martinez and L. M. Liz-Marzan, J. Phys. Chem. Lett., 2011, 2, 846-851.

19 M. L. Nesterov, X. H. Yin, M. Schaferling, H. Giessen and T. Weiss, ACS Photonics, 2016, 3, 578-583.

20 A. O. Govorov and Z. Y. Fan, Chemphyschem, 2012, 13, 2551-2560.

21 B. T. Draine, Astrophys. J., 1988, 333, 848-872.

22 J. Jin, The Finite Element Method in Electromagnetics, Wiley, New York, 2002.

23 G. Zheng, Z. Bao, J. Perez-Juste, R. Du, W. Liu, J. Dai, W. Zhang, L. Y. S. Lee and K. Y. Wong, Angew. Chem. Int. Ed., 2018, 57, 16452-16457.

24 S. Hou, J. Yan, Z. J. Hu and X. C. Wu, Chem. Commun., 2016, 52, 2059-2062.

25 C. L. Hao, L. G. Xu, M. Z. Sun, W. Ma, H. Kuang and C. L. Xu, Adv. Funct. Mater., 2018, 28, 1802372.

26 X. L. Wu, L. G. Xu, W. Ma, L. Q. Liu, H. Kuang, W. J. Yan, L. B. Wang and C. L. Xu, Adv. Funct. Mater., 2015, 25, 850-854.

27 C. L. Hao, L. G. Xu, W. Ma, X. L. Wu, L. B. Wang, H. Kuang and C. L. Xu, Adv. Funct. Mater., 2015, 25, 5816-5822.

28 H. E. Lee, H. Y. Ahn, J. Mun, Y. Y. Lee, M. Kim, N. H. Cho, K. Chang, W. S. Kim, J. Rho and K. T. Nam, Nature, 2018, 556, 360-365.

29 S. Ostovar pour, L. Rocks, K. Faulds, D. Graham, V. Parchaňský, P. Bouř and E. W. Blanch, Nat. Chem., 2015, 7, 591-596.

30 N. H. Cho, G. H. Byun, Y. C. Lim, S. W. Im, H. Kim, H. E. Lee, H. Y. Ahn and K. T. Nam, ACS Nano, 2020, 14, 3595-3602.

31 H. E. Lee, R. M. Kim, H. Y. Ahn, Y. Y. Lee, G. H. Byun, S. W. Im, J. Mun, J. Rho and K. T. Nam, Nat. Commun., 2020, 11, 263.

32 G. Gonzalez-Rubio, J. Mosquera, V. Kumar, A. Pedrazo-Tardajos, P. Llombart, D. M. Solis, I. Lobato, E. G. Noya, A. Guerrero-Martinez, J. M. Taboada, F. Obelleiro, L. G. MacDowell, S. Bals and L. M. Liz-Marzan, Science, 2020, 368, 1472-1477. 
33 M. Nakagawa and T. Kawai, J. Am. Chem. Soc., 2018, 140, 4991-4994.

34 A. Ben-Moshe, S. G. Wolf, M. Bar Sadan, L. Houben, Z. Y. Fan, A. O. Govorov and G. Markovich, Nat. Commun., 2014, 5, 4302.

35 K. Saito and T. Tatsuma, Nano Lett., 2018, 18, 3209-3212.

36 K. Morisawa, T. Ishida and T. Tatsuma, ACS Nano, 2020, 14, 3603-3609.

37 K. M. Mayer and J. H. Hafner, Chem. Rev., 2011, 111, 3828-3857.

38 H. H. Jeong, A. G. Mark, M. Alarcon-Correa, I. Kim, P. Oswald, T. C. Lee and P. Fischer, Nat. Commun., 2016, 7, 11331.

39 W. J. Liu, Z. N. Zhu, K. Deng, Z. T. Li, Y. L. Zhou, H. B. Qu, Y. Gao, S. N. Che and Z. Y. Tang, J. Am. Chem. Soc., 2013, 135, 9659-9664.

40 M. L. Brongersma, N. J. Halas and P. Nordlander, Nat. Nanotechnol., 2015, 10, 25-34.

41 Y. R. Fang, R. Verre, L. Shao, P. Nordlander and M. Kall, Nano Lett., 2016, 16, 51835190.

42 P. Herves, M. Perez-Lorenzo, L. M. Liz-Marzan, J. Dzubiella, Y. Lu and M. Ballauff, Chem. Soc. Rev., 2012, 41, 5577-5587.

43 G. V. Hartland, Chem. Rev., 2011, 111, 3858-3887.

44 F. L. Gao, M. Z. Sun, W. Ma, X. L. Wu, L. Q. Liu, H. Kuang and C. L. Xu, Adv. Mater., 2017, 29, 1606864.

45 M. Kim, J. H. Lee and J. M. Nam, Adv. Sci., 2019, 6, 1900471.

46 A. R. Miandashti, L. K. Khorashad, M. E. Kordesch, A. O. Govorov and H. H. Richardson, ACS Nano, 2020, 14, 4188-4195.

47 I. Dolamic, B. Varnholt and T. Burgi, Nat. Commun., 2015, 6, 7117.

48 J. K. Gansel, M. Thiel, M. S. Rill, M. Decker, K. Bade, V. Saile, G. von Freymann, S. Linden and M. Wegener, Science, 2009, 325, 1513-1515.

49 V. Mujica, Nat. Chem., 2015, 7, 543-544.

50 M. Grzelczak, L. M. Liz-Marzan and R. Klajn, Chem. Soc. Rev., 2019, 48, 1342-1361. 